\title{
THE CARDINALITY OF THE AUGMENTATION CATEGORY OF A LEGENDRIAN LINK
}

\author{
LENHARD NG, DAN RUTHERFORD, VIVEK SHENDE, AND STEVEN SIVEK
}

\begin{abstract}
We introduce a notion of cardinality for the augmentation category associated to a Legendrian knot or link in standard contact $\mathbb{R}^{3}$. This 'homotopy cardinality' is an invariant of the category and allows for a weighted count of augmentations, which we prove to be determined by the ruling polynomial of the link. We present an application to the augmentation category of doubly Lagrangian slice knots.
\end{abstract}

\section{INTRODUCTION}

To a Legendrian knot or link $\Lambda$ in the standard contact $\mathbb{R}^{3}$, one can associate a semi-free noncommutative dga $\mathcal{A}(\Lambda)$, the Chekanov-Eliashberg dga, whose generators are the Reeb chords of $\Lambda$, and whose differential counts certain holomorphic disks in the symplectization [Eli98, Che02, EGH00]. Legendrian isotopies $\Lambda \sim \Lambda^{\prime}$ induce homotopy equivalences $\mathcal{A}(\Lambda) \sim \mathcal{A}\left(\Lambda^{\prime}\right)$. In practice, information is often extracted from $\mathcal{A}(\Lambda)$ by studying its dga maps to a field, called augmentations. For instance, one could just count them: that is, fix a finite field $\mathbb{F}_{q}$ and count dga morphisms $\mathcal{A}(\Lambda) \rightarrow \mathbb{F}_{q}$.

A series of works [CP05, Fuc03, FI04, Sab05, NS06, HR15] gives increasingly precise relationships between this count and certain combinatorial objects called graded normal rulings. A normal ruling of a Legendrian front is a decomposition into boundaries of embedded disks, each containing one right cusp and one left cusp, and satisfying certain additional requirements; see Figure 1 for an example and any of the above references for a more precise definition. The data of a normal ruling is just the specification, at each crossing, of whether the two disk boundaries simply trace out the knot, or 'switch' at the crossing. A normal ruling is graded if all switches occur between strands of equal Maslov potential. The conditions defining graded normal rulings are such that a generating family (or sheaf) for the Legendrian link determines such a ruling by applying Barannikov normal form to the filtered complexes over each $x$ coordinate [Bar94, CP05].

The switching data can be viewed as prescribing a gluing of the disks into a surface, and the Euler characteristic of this surface is a natural invariant of the ruling. For a given Legendrian front, we write $\mathcal{R}$ for the set of graded normal rulings, and $\chi(R)$ for the Euler characteristic of the surface associated to a ruling $R \in \mathcal{R}$; note that $\chi(R)=$ \#right cusps - \#switches. Following [CP05], we can then assemble these into the ruling polynomial $R_{\Lambda}(z)=$ $\sum_{R \in \mathcal{R}} z^{-\chi(R)}$.

To state the relationship between rulings and the count of augmentations, we define the shifted Euler characteristic

$$
\chi_{*}(\Lambda)=\sum_{i \geq 0}(-1)^{i} r_{i}+\sum_{i<0}(-1)^{i+1} r_{i}
$$

as in [NS06], where $r_{i}$ is the number of Reeb chords whose corresponding element in $\mathcal{A}(\Lambda)$ has degree $i$. If $\Lambda$ has $\ell$ components, we now have the following result of Henry and Rutherford [HR15, Remark 3.3(ii)]:

$$
q^{-\chi_{*}(\Lambda) / 2}(q-1)^{-\ell} \cdot \#\left\{\mathcal{A}(\Lambda) \rightarrow \mathbb{F}_{q}\right\}=R_{\Lambda}\left(q^{1 / 2}-q^{-1 / 2}\right) .
$$

There is a certain tension in the above equation. The right hand side of (1) has been proven by Chekanov and Pushkar [CP05] to be an invariant of Legendrian isotopy, by checking Legendrian Reidemeister moves. Moreover, a result of Rutherford [Rut06] asserts that in the 2-periodic setting, the right hand side is in fact a topological invariant, a part of the HOMFLY-PT polynomial of the underlying topological knot.

But on the left hand side, since only the homotopy class of the algebra $\mathcal{A}(\Lambda)$ is an invariant of the Legendrian isotopy class of $\Lambda$, the set of augmentations is not an invariant. However, there is now a unital $A_{\infty}$ category $\mathcal{A} u g_{+}\left(\Lambda ; \mathbb{F}_{q}\right)$, the augmentation category, whose objects are augmentations, and whose endomorphisms are a version of linearized contact cohomology [NRS $\left.{ }^{+} 15\right]$. This category is a Legendrian isotopy invariant of $\Lambda$, in the sense that Legendrian isotopies induce $A_{\infty}$-equivalences of categories. It was introduced in order to match a certain category of sheaves studied in [STZ14]— the subcategory of compactly supported, microlocal rank one objects in the category $S h_{\Lambda}\left(\mathbb{R}^{2} ; \mathbb{F}_{q}\right)$ of 


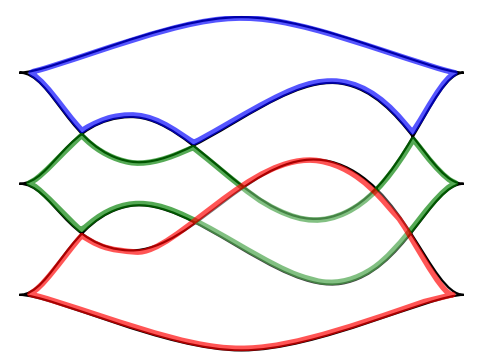

FIGURE 1. A graded normal ruling $R$ of a Legendrian $m\left(8_{21}\right)$ knot with $\chi(R)=-1$.

sheaves on the front plane with microsupport on $\Lambda$. This category of sheaves is in turn equivalent to the full subcategory consisting of objects asymptotic to $\Lambda$ in the derived infinitesimally wrapped Fukaya category [NZ09]. These categories are also known to be equivalent to a category of generating families [She15].

Our goal here is to show that the left hand side of Equation (1) can be replaced by the cardinality, suitably interpreted, of the augmentation category $\mathcal{A} u g_{+}\left(\Lambda ; \mathbb{F}_{q}\right)$.

Already for usual categories, there are two ways to count objects. ${ }^{1}$ The first is to just count the number of isomorphism classes. The second, usually more natural, is to compute the sum over the isomorphism classes of the inverse of the number of automorphisms of an object in the class. The reason to prefer the latter to the former is familiar from the theory of finite group actions on sets. Given a set $S$ carrying the action of a group $G$, one can form the quotient set $S / G$, whose elements are equivalence classes, or one can form the groupoid quotient $[S / G]$ : its objects are the $G$-orbits, and their endomorphisms are given by the stabilizers. One has \# $S / G)=\# S / \# G$ only when the $G$ action is free, while by the orbit-stabilizer theorem, one always has $\#[S / G]=\# S / \# G$.

However, computer experiments have shown that neither counting equivalence classes nor computing the groupoid cardinality of $H^{0}\left(\mathcal{A u g} g_{+}\left(\Lambda ; \mathbb{F}_{q}\right)\right)$ will give the left hand side of Equation (1), even up to multiplication by powers of $q^{1 / 2}$ and $q-1$. For the count of equivalence classes, the Legendrian $m\left(8_{21}\right)$ knot studied in [MS05] (and pictured in Figure 1) has 16 augmentations over $\mathbb{F}_{2}$ which fall into 10 equivalence classes, whereas the left side of Equation (1) is $4 \sqrt{2}$. For the groupoid cardinality, consider the Legendrian $m\left(9_{45}\right)$ knot discussed in [NRS ${ }^{+} 15$, Section 4.4.4]. This has 5 augmentations over $\mathbb{F}_{2}$, none of which are equivalent; three of them have $|\operatorname{Aut}(\epsilon)|=1$ and two have $|\operatorname{Aut}(\epsilon)|=2$, so the groupoid cardinality is 4, whereas the left side of Equation (1) is equal to $5 / \sqrt{2}$.

What does work is the homotopy cardinality [BD01], also sometimes called the multiplicative Euler characteristic. This is a number attached to spaces with finitely many homotopy groups, all of finite cardinality, and is defined as:

$$
\# X:=\sum_{[x] \in \pi_{0}(X)} \frac{\left|\pi_{2}(x, X)\right|\left|\pi_{4}(x, X)\right| \cdots}{\left|\pi_{1}(x, X)\right|\left|\pi_{3}(x, X)\right| \cdots} .
$$

The connection to the previously introduced notion of groupoid cardinality comes from identifying $[S / G]$ with the homotopy-theoretic quotient-i.e., replacing $S$ with a space homotopy equivalent to it on which $G$ acts freely, and then taking the quotient - whereupon $\pi_{0}([S / G])$ is identified with the set of orbits, $\pi_{1}(s,[S / G])$ is identified with the stabilizer of $s$, and all higher $\pi_{i}$ are trivial. One previous use of the notion of homotopy cardinality was to make sense of the Dijkgraaf-Witten theory with a higher groupoid as gauge group [MP07].

To apply this notion here, we take the groupoid part of the augmentation category. That is, we write $\pi_{\geq 0} \mathcal{A} u g_{+}\left(\Lambda ; \mathbb{F}_{q}\right)^{*}$ for the groupoid consisting of isomorphisms in the truncation $\pi_{\geq 0} \mathcal{A} u g_{+}\left(\Lambda ; \mathbb{F}_{q}\right)$. We have cohomological conventions for maps in $\mathcal{A} u g_{+}$, so $\pi_{\geq 0}$ means that we take only morphisms in non-positive degree. The homotopy cardinality of (the classifying space of) this groupoid is then given by the formula:

$$
\# \pi_{\geq 0} \mathcal{A} u g_{+}\left(\Lambda ; \mathbb{F}_{q}\right)^{*}=\sum_{[\epsilon] \in \mathcal{A} u g_{+}\left(\Lambda ; \mathbb{F}_{q}\right) / \sim} \frac{1}{|\operatorname{Aut}(\epsilon)|} \cdot \frac{\left|H^{-1} \operatorname{Hom}(\epsilon, \epsilon)\right| \cdot\left|H^{-3} \operatorname{Hom}(\epsilon, \epsilon)\right| \cdots}{\left|H^{-2} \operatorname{Hom}(\epsilon, \epsilon)\right| \cdot\left|H^{-4} \operatorname{Hom}(\epsilon, \epsilon)\right| \cdots},
$$

where $\operatorname{Aut}(\epsilon)$ is the set of units of $H^{*} \operatorname{Hom}(\epsilon, \epsilon)$. Here we relate this quantity to the naive count of augmentations:

Theorem 1. We have:

$$
\# \pi_{\geq 0} \mathcal{A} u g_{+}\left(\Lambda ; \mathbb{F}_{q}\right)^{*}=q^{\frac{t b-\chi_{*}}{2}} \cdot(q-1)^{-\ell} \cdot \#\left\{\mathcal{A}(\Lambda) \rightarrow \mathbb{F}_{q}\right\}
$$

${ }^{1}$ More properly, we are counting in the groupoid of isomorphisms in the category. 
Remark. This suggests that $\# \pi_{\geq 0} \mathcal{A} u g_{+}\left(\Lambda ; \mathbb{F}_{q}\right)^{*}$ is a quotient of the set $\left\{\mathcal{A}(\Lambda) \rightarrow \mathbb{F}_{q}\right\}$ by some higher groupoid whose cardinality is $q^{\frac{\chi *-t b}{2}} \cdot(q-1)^{\ell}$.

We deduce the following result about counting objects in the sheaf category, conjectured in [STZ14, Conjecture 7.5].

Corollary 2. We have the equalities:

$$
\# \pi_{\geq 0} S h_{\Lambda ; 1}\left(\mathbb{R}^{2} ; \mathbb{F}_{q}\right)^{*}=\# \pi_{\geq 0} \mathcal{A} u g_{+}\left(\Lambda ; \mathbb{F}_{q}\right)^{*}=q^{t b(\Lambda) / 2} R_{\Lambda}\left(q^{1 / 2}-q^{-1 / 2}\right) .
$$

Proof. The first equality holds by [NRS $\left.{ }^{+} 15\right]$, since both sides only depend on the cohomology categories, which are isomorphic. The second equality follows by combining Theorem 1 with Equation (1).

One application of this theorem is a strong restriction on Legendrian knots which we call doubly Lagrangian slice. A theorem of Eliashberg and Polterovich [EP96] (cf. [Cha13]) implies that any Lagrangian concordance from the standard Legendrian unknot $U$ with $t b(U)=-1$ to itself, i.e., a Lagrangian cylinder in $\mathbb{R} \times \mathbb{R}^{3}$ which agrees with the product $\mathbb{R} \times U$ outside a compact set, can in fact be identified with $\mathbb{R} \times U$ by a compactly supported Hamiltonian isotopy. It is possible, however, that for some nontrivial Legendrian $\Lambda$ there are Lagrangian concordances from $U$ to $\Lambda$ and from $\Lambda$ to $U$-in other words, that a Lagrangian concordance $L$ from $U$ to itself might satisfy

$$
L \cap\left((-\epsilon, \epsilon) \times \mathbb{R}^{3}\right)=(-\epsilon, \epsilon) \times \Lambda
$$

for some $\epsilon>0$-and in this case we say that $\Lambda$ is doubly Lagrangian slice. In Section 3 we prove the following.

Theorem 3. If $\Lambda$ is doubly Lagrangian slice, then there is an $A_{\infty}$ quasi-equivalence $\mathcal{A} u g_{+}\left(\Lambda ; \mathbb{F}_{q}\right) \cong \mathcal{A} u g_{+}\left(U ; \mathbb{F}_{q}\right)$ over any finite field $\mathbb{F}_{q}$.

Given an augmentation $\epsilon: \mathcal{A}(\Lambda) \rightarrow \mathbb{F}_{q}$, one can complete $\mathcal{A}(\Lambda)$ to the $I$-adic completion $\widehat{\mathcal{A}}(\Lambda)$, where $I$ is the ideal ker $\epsilon \subset \mathcal{A}(\Lambda)$. Using the above, we also prove that there is a quasi-isomorphism $\widehat{\mathcal{A}}(\Lambda) \rightarrow \widehat{\mathcal{A}}(U)$, where $\widehat{\mathcal{A}}(U)$ is easily computed to be the power series ring $\mathbb{F}_{q}[[a]]$ with trivial differential, whenever $\Lambda$ is doubly Lagrangian slice. See Proposition 15 for details.

So far, our discussion has been for augmentations and rulings with a full $\mathbb{Z}$ grading. One can also consider rulings and the augmentation category graded in $\mathbb{Z} /(2 m)$ for some $m \in \mathbb{Z}$, where as mentioned earlier the $m=1$ case is related to the HOMFLY-PT polynomial. The complexes in $\mathcal{A} u g_{+}\left(\Lambda ; \mathbb{F}_{q}\right)$ are now $2 m$-periodic. It is then unclear what should substitute for the homotopy cardinality $\# \pi_{\geq 0} \mathcal{A} u g_{+}\left(\Lambda ; \mathbb{F}_{q}\right)^{*}$, but we provide a candidate in Corollary 20 that we conjecture is related to the $2 m$-graded ruling polynomial of $\Lambda$ in the same way as Theorem 1 . For the 2-periodic case, for example, we expect the homotopy cardinality to be given by

$$
\sum_{[\epsilon] \in \mathcal{A} u g_{+}\left(\Lambda ; \mathbb{F}_{q}\right) / \sim} \frac{1}{|\operatorname{Aut}(\epsilon)|}\left|H^{\mathrm{ev}} \operatorname{Hom}(\epsilon, \epsilon)\right|^{1 / 2} q^{-\ell / 2},
$$

where $H^{\mathrm{ev}}$ denotes cohomology in even degree.

In Section 2, we prove our main result, Theorem 1. We discuss the application to doubly Lagrangian slice knots in Section 3, and the $\mathbb{Z} /(2 m)$-graded setting in Section 4.

Acknowledgments. We would like to thank the American Institute of Mathematics for sponsoring a SQuaRE group, "Sheaf theory and Legendrian knots", at whose 2015 meeting we began the work for this paper. We are also grateful to the other participants of the SQuaRE, David Treumann, Harold Williams, and Eric Zaslow, for helpful conversations. We also thank André Henriques, Brad Henry, and Qiaochu Yuan. The work of LN was supported by NSF grant DMS1406371 and a grant from the Simons Foundation (\#341289 to Lenhard Ng). The work of VS was supported by NSF grant DMS-1406871 and a Sloan fellowship. The work of SS was supported by NSF grant DMS-1506157.

\section{Counting}

We turn to the proof of Theorem 1. Throughout this section, we consider augmentations with values in a field $\mathbb{k}$, which will be assumed to be a finite field $\mathbb{F}_{q}$ when counting is necessary. The basic idea to prove Theorem 1 is to establish a correspondence between the (not necessarily closed) degree zero automorphisms of a given augmentation on the one hand, and isomorphic augmentations on the other. The correspondence will be many to one, but the discrepancy will be accounted for by homotopies, higher homotopies, etc. 
The starting point is the comparison between morphisms in the augmentation category and dga homotopies. Recall that if $f, f^{\prime}: \mathcal{A} \rightarrow \mathcal{B}$ are dga morphisms, then a chain homotopy between them, i.e., a map $h: \mathcal{A} \rightarrow \mathcal{B}$ with $d_{\mathcal{B}} h \pm h d_{\mathcal{A}}=f-f^{\prime}$, is a dga homotopy between them if $h$ is an $\left(f, f^{\prime}\right)$-derivation, i.e., $h(a b)=f(a) h(b) \pm h(a) f^{\prime}(b)$.

Recall also from $\left[\mathrm{NRS}^{+} 15\right]$ that in the case where $\Lambda$ is a knot, if Hom spaces of the augmentation category are calculated by perturbing the 2-copy via a Morse function on the knot with a single pair of critical points (the "Lagrangian projection perturbation"), then each $\operatorname{Hom}\left(\epsilon, \epsilon^{\prime}\right)$ is generated as a module by certain terms $a_{i}^{+}$corresponding to the Reeb chords of the original knot, together with a degree zero element $y^{+}$coming from one of the critical points of the Morse function, and a degree one element $x^{+}$coming from the other. The element $-y^{+}$is a strict identity on self-hom spaces, which we write as $e_{\epsilon} \in \operatorname{Hom}(\epsilon, \epsilon)$. Note that the degree of the generator of the Hom space corresponding to a Reeb chord is 1 greater than the degree of the corresponding generator of the dga. There is a precise relation between dga homotopies and closed isomorphisms in $\mathcal{A} u g_{+}(\Lambda)$ :

Proposition 4 ([NRS ${ }^{+}$15, Proposition 5.16]). Every general element of $\operatorname{Hom}^{0}\left(\epsilon, \epsilon^{\prime}\right)$ of the form

$$
\alpha:=-y^{+}-\sum k_{i} a_{i}^{+}
$$

is invertible, meaning that there are elements $\beta, \gamma \in \operatorname{Hom}^{0}\left(\epsilon^{\prime}, \epsilon\right)$ such that $m_{2}(\beta, \alpha)=e_{\epsilon}$ and $m_{2}(\alpha, \gamma)=e_{\epsilon^{\prime}}$. Moreover, we have $m_{1}(\alpha)=0$ if and only if the $\left(\epsilon, \epsilon^{\prime}\right)$-derivation $K: \mathcal{A}(\Lambda) \rightarrow \mathbb{k}$ defined by requiring $K\left(a_{i}\right)=k_{i}$ for each $i$ is a dga homotopy from $\epsilon$ to $\epsilon^{\prime}$.

If $\Lambda$ is a link with $\ell$ components, labeled $1,2, \ldots, \ell$, we define a function $r \times c$ on the set of Reeb chords such that $r\left(a_{i}\right)$ and $c\left(a_{i}\right)$ are the labels of the overcrossing and undercrossing strands of $\Lambda$ at the chord $a_{i}$. (This is called a link grading in [NRS $\left.{ }^{+} 15\right]$.) We assume that $\Lambda$ has a single base point on each component, and the generators $t_{i}^{ \pm 1}$ of $\mathcal{A}(\Lambda)$ correspond to the base point on component $i$; we will write $r\left(t_{i}^{ \pm 1}\right)=c\left(t_{i}^{ \pm 1}\right)=i$. In this case each Hom $\left(\epsilon, \epsilon^{\prime}\right)$ will have generators $y_{1}^{+}, \ldots, y_{\ell}^{+}$in degree zero and $x_{1}^{+}, \ldots, x_{\ell}^{+}$in degree one, corresponding to the minima and maxima of a Morse function on $\Lambda$ by which we perturb each component, in addition to the Reeb chord generators $a_{i}^{+}$.

In the case where $\Lambda$ has multiple components, the characterization of invertible elements and isomorphisms in $\mathcal{A} u g_{+}(\Lambda)$ closely resembles the one described in Proposition 4. We will explain this below, showing in Proposition 7 that the (not necessarily closed) automorphisms $\alpha \in \operatorname{Hom}^{0}(\epsilon, \epsilon)$, including those for which $m_{1}(\alpha) \neq 0$, can instead be viewed as (closed) isomorphisms from $\epsilon$ to some uniquely determined $\epsilon^{\prime}$.

Lemma 5. For fixed $\epsilon$, and arbitrarily chosen $k_{i} \in \mathbb{k}$, there is a unique augmentation $\epsilon^{\prime}: \mathcal{A} \rightarrow \mathbb{k}$ and a unique $\left(\epsilon, \epsilon^{\prime}\right)$-derivation $K$ such that $\epsilon^{\prime}\left(t_{i}\right)=\epsilon\left(t_{i}\right)$ for $i=1, \ldots, \ell ; K\left(a_{i}\right)=k_{i}$ for all $a_{i}$; and $\epsilon-\epsilon^{\prime}=K \circ \partial$.

Proof. We make use of the height filtration on the dga, originally due to Chekanov [Che02], which is defined using the lengths of the Reeb chords: if $a_{i}$ has height $h\left(a_{i}\right)$, and the differential counts a disk $u$ with positive end at $a_{0}$ and negative ends at $b_{1}, b_{2}, \ldots, b_{m}$, then $h\left(a_{0}\right)>\sum h\left(b_{i}\right)$, since by Stokes's theorem their difference is the area of the projection $\pi_{x y}(u)$. Thus if the Reeb chords $a_{1}, \ldots, a_{r}$ are ordered by increasing height, we have $\partial a_{i} \in F_{i-1} \mathcal{A}:=$ $\mathbb{k}\left\langle a_{1}, \ldots, a_{i-1}\right\rangle$. We will define $\epsilon^{\prime}$ inductively by height.

Suppose that $\epsilon^{\prime}$ has been defined on $F_{i-1} \mathcal{A}$ so that on $F_{i-1} \mathcal{A}$ we have $\epsilon^{\prime} \circ \partial=0$ and $\epsilon-\epsilon^{\prime}=K \circ \partial$, where $K$ is the extension of the map $a_{i} \mapsto k_{i}$ to $F_{i-1} \mathcal{A}$ as an $\left(\epsilon, \epsilon^{\prime}\right)$-derivation. Then we set $\epsilon^{\prime}\left(a_{i}\right):=\epsilon\left(a_{i}\right)-K\left(\partial a_{i}\right)$ and extend $\epsilon^{\prime}$ to $F_{i} \mathcal{A}$ as an algebra homomorphism, and we can extend $K$ to $F_{i} \mathcal{A}$ as an $\left(\epsilon, \epsilon^{\prime}\right)$-derivation satisfying $K\left(a_{i}\right)=k_{i}$. We need to check that (i) $\epsilon^{\prime}:\left(F_{i} \mathcal{A}, \partial\right) \rightarrow(\mathbb{k}, 0)$ is a chain map, and (ii) $\epsilon-\epsilon^{\prime}=K \circ \partial$ continues to hold on $F_{i} \mathcal{A}$. Note that the identities $\epsilon^{\prime} \circ \partial_{\mathcal{A}}=\partial_{\mathbb{k}} \circ \epsilon^{\prime}=0$ and $\epsilon-\epsilon^{\prime}=\partial_{\mathbb{k}} \circ K+K \circ \partial_{\mathcal{A}}=K \circ \partial_{\mathcal{A}}$ will be satisfied by the algebra maps $\epsilon, \epsilon^{\prime}: \mathcal{A} \rightarrow \mathbb{k}$ and the $\left(\epsilon, \epsilon^{\prime}\right)$-derivation $K$ if they are satisfied on all of the generators of $\mathcal{A}$.

To verify (i), since $\partial a_{i} \in F_{i-1} \mathcal{A}$, the inductive hypothesis gives

$$
\epsilon^{\prime}\left(\partial a_{i}\right)=\epsilon\left(\partial a_{i}\right)-K \circ \partial\left(\partial a_{i}\right)=0 .
$$

For (ii), the identity holds by construction when applied to $a_{i}$, so it holds on all of $F_{i} \mathcal{A}$.

Lemma 6. If $\epsilon: \mathcal{A}(\Lambda) \rightarrow \mathbb{k}$ is an augmentation, then for any invertible elements $d_{1}, \ldots, d_{\ell} \in \mathbb{k}^{\times}$, the algebra map $\epsilon^{\prime}: \mathcal{A}(\Lambda) \rightarrow \mathbb{k}$ defined on generators by

$$
\epsilon^{\prime}\left(a_{i}\right)=\frac{d_{r\left(a_{i}\right)}}{d_{c\left(a_{i}\right)}} \epsilon\left(a_{i}\right)
$$

is also an augmentation. 
Proof. We need to check that $\epsilon^{\prime}\left(\partial a_{i}\right)=0$ for each generator $a_{i}$ given that $\epsilon\left(\partial a_{i}\right)=0$. The differential $\partial a_{i}$ counts disks $u$ with positive end at $a_{i}$ and negative ends at Reeb chords $b_{1}, \ldots, b_{m}$, and such disks contribute words $w(u)$ of the form $\pm b_{1} \ldots b_{m}$, possibly with $t_{i}^{ \pm 1}$ generators sprinkled throughout, to $\partial a_{i}$. It follows that

$$
\epsilon^{\prime}(w(u))=\frac{d_{r\left(b_{1}\right)}}{d_{c\left(b_{1}\right)}} \ldots \frac{d_{r\left(b_{m}\right)}}{d_{c\left(b_{m}\right)}} \epsilon(w(u)) .
$$

But we also know that $r\left(a_{i}\right)=r\left(b_{1}\right)$ and $c\left(a_{i}\right)=c\left(b_{m}\right)$, and that $c\left(b_{j}\right)=r\left(b_{j+1}\right)$ for $1 \leq j \leq m-1$, so this simplifies to $\epsilon^{\prime}(w(u))=\frac{d_{r\left(a_{i}\right)}}{d_{c\left(a_{i}\right)}} \cdot \epsilon(w(u))$. Summing over all $u$, we have $\epsilon^{\prime}\left(\partial a_{i}\right)=\frac{d_{r\left(a_{i}\right)}}{d_{c\left(a_{i}\right)}} \cdot \epsilon\left(\partial a_{i}\right)=0$.

According to $\left[\mathrm{NRS}^{+} 15\right.$, Proposition 3.28], the category $\mathcal{A} u g_{+}(\Lambda)$ is strictly unital, with unit $e_{\epsilon}=-\sum_{i=1}^{\ell} y_{i}^{+}$in each $\operatorname{Hom}(\epsilon, \epsilon)$. Given elements $\alpha \in \operatorname{Hom}^{0}\left(\epsilon_{1}, \epsilon_{2}\right)$ and $\beta, \gamma \in \operatorname{Hom}^{0}\left(\epsilon_{2}, \epsilon_{1}\right)$, we can compute the products $m_{2}(\beta, \alpha)$ and $m_{2}(\alpha, \gamma)$ from [NRS ${ }^{+} 15$, Proposition 4.14], which determines the dga of the 3-copy of $\Lambda$, and [NRS ${ }^{+} 15$, Definition 3.16], which produces the $A_{\infty}$ operations from the sequence of $n$-copy dgas. In particular, if $m_{2}(\beta, \alpha)=e_{\epsilon_{1}}$ then the coefficients $d_{i}^{\alpha}$ and $d_{i}^{\beta}$ of each $y_{i}^{+}$in $\alpha$ and $\beta$ respectively must satisfy $d_{i}^{\alpha} \cdot d_{i}^{\beta}=1$, and likewise if $m_{2}(\alpha, \gamma)=e_{\epsilon_{2}}$ then $d_{i}^{\alpha} \cdot d_{i}^{\gamma}=1$. Moreover, given $\alpha$ with $d_{i}^{\alpha} \in \mathbb{k}^{\times}$, we can repeat the argument of [NRS ${ }^{+} 15$, Proposition 5.17] to show that there are in fact some elements $\beta$ and $\gamma$ such that $m_{2}(\beta, \alpha)=e_{\epsilon_{1}}$ and $m_{2}(\alpha, \gamma)=e_{\epsilon_{2}}$, and that if $m_{1}(\alpha)=0$ then $m_{1}(\beta)=m_{1}(\gamma)=0$ and $[\beta]=[\gamma]$ in $H^{0} \operatorname{Hom}\left(\epsilon_{2}, \epsilon_{1}\right)$ as well. (The construction of $\beta$ and $\gamma$ proceeds once again by induction on height once we replace the $m_{2}$ computations of [NRS ${ }^{+} 15$, Proposition 5.15] with the more general $m_{2}\left(y_{i}^{+}, a_{j}^{+}\right)=-\delta_{i, c\left(a_{j}\right)} a_{j}^{+}$and $m_{2}\left(a_{j}^{+}, y_{i}^{+}\right)=-\delta_{i, r\left(a_{j}\right)} a_{j}^{+}$.)

Proposition 7. Let $\Lambda$ be an $\ell$-component Legendrian link with one base point on each component. Given an augmentation $\epsilon_{1}: \mathcal{A}(\Lambda) \rightarrow \mathbb{k}$ and a degree-zero element of the form

$$
\alpha=d_{1} y_{1}^{+}+\cdots+d_{\ell} y_{\ell}^{+}+\sum_{j=1}^{r} k_{j} a_{j}^{+},
$$

where $d_{i} \in \mathbb{k}^{\times}$for all $i$ and $k_{j} \in \mathbb{k}$ for all $j$, there is a unique augmentation $\epsilon_{2}: \mathcal{A}(\Lambda) \rightarrow \mathbb{k}$ such that $\alpha$ is an isomorphism in $\operatorname{Hom}^{0}\left(\epsilon_{1}, \epsilon_{2}\right)$.

Proof. Given an augmentation $\epsilon_{2}$, we must determine whether $m_{1}(\alpha)=0$ in $\operatorname{Hom}\left(\epsilon_{1}, \epsilon_{2}\right)$, and if this is the case then it will follow from the above discussion that $\alpha$ is an isomorphism. In what follows, we will let $\epsilon^{\prime}: \mathcal{A} \rightarrow \mathbb{k}$ denote the algebra homomorphism defined on generators by $\epsilon^{\prime}\left(a_{j}\right)=\frac{d_{r\left(a_{j}\right)}}{d_{c\left(a_{j}\right)}} \epsilon_{2}\left(a_{j}\right)$ and $\epsilon^{\prime}\left(t_{i}\right)=\epsilon_{2}\left(t_{i}\right)$; this is an augmentation by Lemma 6 . We will also let $K$ denote the unique $\left(\epsilon_{1}, \epsilon^{\prime}\right)$-derivation defined on generators by $K\left(a_{j}\right)=\frac{k_{j}}{d_{c\left(a_{j}\right)}}$.

The computation of $m_{1}(\alpha)$ involves essentially the same computations as in [NRS ${ }^{+} 15$, Proposition 5.16]. We compute $m_{1}$ according to [NRS ${ }^{+} 15$, Proposition 4.14] and [NRS ${ }^{+} 15$, Definition 3.16]:

$$
\begin{aligned}
& m_{1}\left(y_{i}^{+}\right)=\left(\epsilon_{1}\left(t_{i}\right)^{-1} \epsilon_{2}\left(t_{i}\right)-1\right) x_{i}^{+}+\sum_{r\left(a_{j}\right)=i} \epsilon_{2}\left(a_{j}\right) \cdot a_{j}^{+}-\sum_{c\left(a_{j}\right)=i}(-1)^{\left|a_{j}\right|} \epsilon_{1}\left(a_{j}\right) \cdot a_{j}^{+}, \\
& m_{1}\left(a_{j}^{+}\right)=\sum_{\substack{a_{k}, b_{1}, \ldots, b_{m} \\
u \in \Delta\left(a_{k} ; b_{1}, \ldots, b_{m}\right)}} \sum_{\substack{1 \leq l \leq n \\
b_{l}=a_{j}}} \sigma_{u} \epsilon_{1}\left(b_{1} \ldots b_{l-1}\right) \epsilon_{2}\left(b_{l+1} \ldots b_{m}\right) a_{k}^{+},
\end{aligned}
$$

where $\Delta\left(a_{j} ; b_{1}, \ldots, b_{m}\right)$ denotes the moduli space of disks which are counted in $\partial a_{j}$ (i.e. rigid holomorphic disks with positive end at $a_{j}$ and negative ends and base points at $\left.b_{1}, \ldots, b_{m}\right)$ and $\sigma_{u}$ is the sign associated to the disk $u$. We also note that $(-1)^{\left|a_{j}\right|} \epsilon_{1}\left(a_{j}\right)=\epsilon_{1}\left(a_{j}\right)$, since $\epsilon\left(a_{j}\right)=0$ unless $\left|a_{j}\right|=0$. Combining these formulas, we have

$$
\begin{aligned}
m_{1}(\alpha)=\sum_{i=1}^{\ell}\left(\epsilon_{1}\left(t_{i}\right)^{-1} \epsilon_{2}\left(t_{i}\right)-1\right) x_{i}^{+}+\sum_{j=1}^{r}\left(d_{r\left(a_{j}\right)} \epsilon_{2}\left(a_{j}\right)-d_{c\left(a_{j}\right)} \epsilon_{1}\left(a_{j}\right)\right) a_{j}^{+} \\
\quad+\sum_{j=1}^{r}\left(\sum_{u \in \Delta\left(a_{j} ; b_{1}, \ldots, b_{m}\right)} \sum_{\substack{1 \leq l \leq m \\
b_{l} \neq t_{i}^{ \pm 1}}} \sigma_{u} \epsilon_{1}\left(b_{1} \ldots b_{l-1}\right) k_{b_{l}} \epsilon_{2}\left(b_{l+1} \ldots b_{m}\right)\right) a_{j}^{+} .
\end{aligned}
$$

In the last sum, we use $k_{b_{l}}$ to denote the coefficient $k_{i}$ such that $a_{i}=b_{l}$. 
The above formula shows that we cannot have $m_{1}(\alpha)=0$ unless $\epsilon_{1}\left(t_{i}\right)=\epsilon_{2}\left(t_{i}\right)$ for all $i$. In order to determine when the coefficient of $a_{j}^{+}$vanishes, we examine the individual terms in the sum over disks $u$ and observe that

$$
\begin{aligned}
k_{b_{l}} \epsilon_{2}\left(b_{l+1} \ldots b_{m}\right) & =\frac{k_{b_{l}}}{d_{c\left(b_{l}\right)}} \cdot\left(\frac{d_{r\left(b_{l+1}\right)}}{d_{c\left(b_{l+1}\right)}} \epsilon_{2}\left(b_{l+1}\right)\right) \cdot \ldots \cdot\left(\frac{d_{r\left(b_{m}\right)}}{d_{c\left(b_{m}\right)}} \epsilon_{2}\left(b_{m}\right)\right) \cdot d_{c\left(a_{j}\right)} \\
& =K\left(b_{l}\right) \epsilon^{\prime}\left(b_{l+1} \ldots b_{m}\right) \cdot d_{c\left(a_{j}\right)},
\end{aligned}
$$

since as in the proof of Lemma 6 we have $c\left(b_{i}\right)=r\left(b_{i+1}\right)$ for all $i<m$ and $c\left(b_{m}\right)=c\left(a_{j}\right)$. Thus $m_{1}(\alpha)$ has $a_{j}^{+}$-coefficient equal to

$$
d_{r\left(a_{j}\right)} \epsilon_{2}\left(a_{j}\right)-d_{c\left(a_{j}\right)} \epsilon_{1}\left(a_{j}\right)+\sum_{u, l} \sigma(u) \epsilon_{1}\left(b_{1} \ldots b_{l-1}\right) K\left(b_{l}\right) \epsilon^{\prime}\left(b_{l+1} \ldots b_{m}\right) \cdot d_{c\left(a_{j}\right)},
$$

which is precisely $d_{c\left(a_{j}\right)}\left(\epsilon^{\prime}\left(a_{j}\right)-\epsilon_{1}\left(a_{j}\right)+K\left(\partial a_{j}\right)\right)$. We conclude that $m_{1}(\alpha)=0$ in $\operatorname{Hom}\left(\epsilon_{1}, \epsilon_{2}\right)$ exactly when $\epsilon_{1}-\epsilon^{\prime}=K \circ \partial$, since this must be satisfied for all generators $a_{j}$ and $K$ is an $\left(\epsilon_{1}, \epsilon^{\prime}\right)$-derivation. But Lemma 5 says that $\epsilon_{1}$ and the values $\frac{k_{j}}{d_{c\left(a_{j}\right)}}$ uniquely determine $K$ and $\epsilon^{\prime}$, and in turn $\epsilon^{\prime}$ uniquely determines $\epsilon_{2}$, so it follows that there is exactly one choice of $\epsilon_{2}$ such that $\alpha$ is a cocycle in $\operatorname{Hom}\left(\epsilon_{1}, \epsilon_{2}\right)$.

Corollary 8. For any $\epsilon \in \mathcal{A} u g_{+}\left(\Lambda ; \mathbb{F}_{q}\right)$, the set

$$
\bigsqcup_{\epsilon^{\prime} \in \mathcal{A u g}_{+}(\Lambda)}\left\{\alpha \in \operatorname{Hom}^{0}\left(\epsilon, \epsilon^{\prime}\right) \mid m_{1}(\alpha)=0 \text {, and }[\alpha] \text { is an isomorphism in } H^{0}\left(\operatorname{Hom}\left(\epsilon, \epsilon^{\prime}\right)\right)\right\}
$$

has cardinality $(q-1)^{\ell} q^{r^{\prime}}$, where $\ell$ denotes the number of components of $\Lambda$ and $r^{\prime}$ is the number of Reeb chords of $\Lambda$ with dga degree -1 .

Proof. Any element of $\operatorname{Hom}^{0}\left(\epsilon, \epsilon^{\prime}\right)$ which represents an invertible class in cohomology must have the form $\sum d_{i} y_{i}^{+}+\beta$ with $d_{1}, \ldots, d_{\ell} \in \mathbb{F}_{q}^{\times}$and $\beta \in \mathbb{F}_{q}\left\{a_{1}^{+}, \ldots, a_{r^{\prime}}^{+}\right\}$, where the $a_{i}$ are the Reeb chords of degree -1 . Thus, for any of the $(q-1)^{\ell}$ choices for $d_{1}, \ldots, d_{\ell}$ we have $q^{r^{\prime}}$ isomorphisms from $\epsilon$ to some $\epsilon^{\prime}$ of the form $\alpha=\sum d_{i} y_{i}^{+}+\beta$, with $\epsilon^{\prime}$ uniquely determined by $\alpha$.

Suppose that the Reeb chords of $\Lambda$ are sorted by height, $h\left(a_{1}\right)<h\left(a_{2}\right)<\cdots<h\left(a_{r}\right)$, and for any $\epsilon_{1}, \epsilon_{2} \in$ $\mathcal{A} u g_{+}(\Lambda ; \mathbb{k})$, consider the descending filtration of

$$
\operatorname{Hom}\left(\epsilon_{1}, \epsilon_{2}\right)=\operatorname{Span}_{\mathrm{k}}\left\{y_{1}^{+}, \ldots, y_{\ell}^{+}, x_{1}^{+}, \ldots, x_{\ell}^{+}, a_{1}^{+}, \ldots, a_{r}^{+}\right\}
$$

given by

$$
\begin{aligned}
F^{-1} \operatorname{Hom}\left(\epsilon_{1}, \epsilon_{2}\right) & =\operatorname{Hom}\left(\epsilon_{1}, \epsilon_{2}\right) \\
F^{0} \operatorname{Hom}\left(\epsilon_{1}, \epsilon_{2}\right) & =\operatorname{Span}_{\mathrm{k}}\left\{x_{1}^{+}, \ldots, x_{\ell}^{+}, a_{1}^{+}, \ldots, a_{r}^{+}\right\} \\
F^{i} \operatorname{Hom}\left(\epsilon_{1}, \epsilon_{2}\right) & =\operatorname{Span}_{\mathrm{k}}\left\{a_{i}^{+}, a_{i+1}^{+}, \ldots, a_{r}^{+}\right\}, \quad \text { for } 1 \leq i \leq r .
\end{aligned}
$$

We claim that the $A_{\infty}$ operations on $\mathcal{A} u g_{+}(\Lambda ; \mathbb{k})$ respect this filtration in the following precise sense.

Proposition 9. For any $k \geq 1$, any augmentations $\epsilon_{1}, \ldots, \epsilon_{k+1}$, and any integers $-1 \leq i_{1}, \ldots, i_{k} \leq r$, we have

$$
m_{k}\left(F^{i_{k}} \operatorname{Hom}\left(\epsilon_{k}, \epsilon_{k+1}\right) \otimes \cdots \otimes F^{i_{1}} \operatorname{Hom}\left(\epsilon_{1}, \epsilon_{2}\right)\right) \subset F^{I} \operatorname{Hom}\left(\epsilon_{1}, \epsilon_{k+1}\right)
$$

where $I= \begin{cases}\max \left\{i_{1}, \ldots, i_{k}\right\}+1, & k \neq 2, \\ \max \left\{i_{1}, i_{2}\right\}, & k=2 .\end{cases}$

Proof. We review relevant aspects of the definition of $m_{k}$; see $\left[\mathrm{NRS}^{+} 15\right.$, Section 3].

The operations $m_{k}$ are constructed from the Chekanov-Eliashberg algebras of $\Lambda$ and of the Lagrangian projection $(k+1)$-copy $\Lambda^{(k+1)}$ (see $\left[\mathrm{NRS}^{+} 15\right.$, Section 4.2.2]) of $\Lambda$. We consider the pure augmentation

$$
\epsilon=\left(\epsilon_{1}, \ldots, \epsilon_{k+1}\right):\left(\mathcal{A}\left(\Lambda^{(k+1)}\right), \partial\right) \rightarrow(\mathbb{k}, 0),
$$

which produces a twisted differential $\partial^{\epsilon}$ on $\mathcal{A}\left(\Lambda^{(k+1)}\right)^{\epsilon}$, where $\mathcal{A}\left(\Lambda^{(k+1)}\right)^{\epsilon}$ is now an algebra over $\mathbb{k}$ freely generated by Reeb chords of $\Lambda^{(k+1)}$. The Reeb chords of $\Lambda^{(k+1)}$ are enumerated as follows: each Reeb chord $a_{l}$ of $\Lambda$ produces Reeb chords $a_{l}^{i j}$ of $\Lambda^{(k+1)}$, where $1 \leq i, j \leq k+1$, and the remaining Reeb chords of $\Lambda^{(k+1)}$ have the form $x_{l}^{i j}$ and 
$y_{l}^{i j}$ for $1 \leq i<j \leq k+1$ and $1 \leq l \leq \ell$. Moreover, we note that the construction of $\Lambda^{(k+1)}$ can be carried out so that we have height inequalities

$$
h\left(y_{l_{1}}\right)<h\left(x_{l_{2}}\right)<h\left(a_{1}\right)<\ldots<h\left(a_{r}\right)
$$

for all $1 \leq l_{1}, l_{2} \leq \ell$ and for any choice of superscripts (omitted from notation). There is a corresponding increasing filtration of $\mathcal{A}\left(\Lambda^{(k+1)}\right)^{\epsilon}$,

$$
F_{-1} \mathcal{A}\left(\Lambda^{(k+1)}\right)^{\epsilon} \subset F_{0} \mathcal{A}\left(\Lambda^{(k+1)}\right)^{\epsilon} \subset F_{1} \mathcal{A}\left(\Lambda^{(k+1)}\right)^{\epsilon} \subset \cdots \subset F_{r} \mathcal{A}\left(\Lambda^{(k+1)}\right)^{\epsilon},
$$

where $F_{-1} \mathcal{A}\left(\Lambda^{(k+1)}\right)^{\epsilon}$ is the sub-algebra generated by elements of the form $y_{l}^{i j}$; we add all generators of the form $x_{l}^{i j}$ to obtain $F_{0} \mathcal{A}\left(\Lambda^{(k+1)}\right)^{\epsilon}$, and then to obtain $F_{p} \mathcal{A}\left(\Lambda^{(k+1)}\right)^{\epsilon}$ from $F_{p-1} \mathcal{A}\left(\Lambda^{(k+1)}\right)^{\epsilon}$, for $1 \leq p \leq r$, we add all generators of the form $a_{p}^{i j}$. As discussed above, the inequalities (2) give $\partial^{\epsilon}\left(F_{p} \mathcal{A}\left(\Lambda^{(k+1)}\right)^{\epsilon}\right) \subset F_{p} \mathcal{A}\left(\Lambda^{(k+1)}\right)^{\epsilon}$ for all $p \geq-1$.

Suppose we wish to determine a composition of the form $m_{k}\left(s_{k}^{+}, \ldots, s_{1}^{+}\right)$, where each $s_{i}^{+} \in \operatorname{Hom}\left(\epsilon_{i}, \epsilon_{i+1}\right)$ is a generator of the form $y_{l}^{+}, x_{l}^{+}$, or $a_{l}^{+}$for some $l$. There are corresponding Reeb chord generators $s_{1}^{12}, s_{2}^{23}, \ldots, s_{k}^{k, k+1}$ in the Chekanov-Eliashberg algebra $\left(\mathcal{A}^{(k+1)}, \partial\right)$ of the $(k+1)$-copy $\Lambda^{(k+1)}$, and we have

$$
\begin{aligned}
m_{k}\left(s_{k}^{+}, \ldots, s_{1}^{+}\right)=(-1)^{\sigma} & \left(\sum_{i=1}^{\ell} y_{i}^{+} \cdot \operatorname{Coeff}_{s_{1}^{12} \cdots s_{k}^{k, k+1}}\left(\partial^{\epsilon} y_{i}^{1, k+1}\right)+\sum_{i=1}^{\ell} x_{i}^{+} \cdot \operatorname{Coeff}_{s_{1}^{12} \cdots s_{k}^{k, k+1}}\left(\partial^{\epsilon} x_{i}^{1, k+1}\right)\right. \\
& \left.+\sum_{i=1}^{r} a_{i}^{+} \cdot \operatorname{Coeff}_{s_{1}^{12} \cdots s_{k}^{k, k+1}}\left(\partial^{\epsilon} a_{i}^{1, k+1}\right)\right)
\end{aligned}
$$

for an appropriate sign $(-1)^{\sigma}$. Showing that $\partial^{\epsilon}$ preserves the filtration (3) translates immediately to showing that

$$
m_{k}\left(F^{i_{k}} \operatorname{Hom}\left(\epsilon_{k}, \epsilon_{k+1}\right) \otimes \cdots \otimes F^{i_{1}} \operatorname{Hom}\left(\epsilon_{1}, \epsilon_{2}\right)\right) \subset F^{J} \operatorname{Hom}\left(\epsilon_{1}, \epsilon_{k+1}\right)
$$

with $J=\max \left\{i_{1}, \ldots, i_{k}\right\}$.

To improve this result when $k \neq 2$, given a generator $s^{1, k+1} \in F_{p} \mathcal{A}\left(\Lambda^{(k+1)}\right)^{\epsilon}$, we need to examine those terms in $\partial^{\epsilon} s^{1, k+1}$ which belong to $F_{p} \mathcal{A}\left(\Lambda^{(k+1)}\right)^{\epsilon} \backslash F_{p-1} \mathcal{A}\left(\Lambda^{(k+1)}\right)^{\epsilon}$. An explicit computation of $\left(\mathcal{A}\left(\Lambda^{(k+1)}\right), \partial\right)$ in terms of $(\mathcal{A}(\Lambda), \partial)$ is given in $\left[\mathrm{NRS}^{+} 15\right.$, Proposition 3.25]. Examining it, we see that the only relevant terms are

$$
\begin{aligned}
\partial Y_{l} & =\left(Y_{l}\right)^{2} \\
\partial X_{l} & =\Delta_{l}^{-1} Y_{l} \Delta_{l} X_{l}-X_{l} Y_{l} \\
\partial A_{k} & =Y_{r(k)} A_{k}-(-1)^{\left|a_{k}\right|} A_{k} Y_{c(k)}+\ldots,
\end{aligned}
$$

where generators $y_{l}^{i j}, x_{l}^{i j}$, and $a_{k}^{i j}$ are placed into matrices $Y_{l}, X_{l}$ and $A_{k} ; r(k)$ and $c(k)$ denote the components of the upper and lower endpoint of the Reeb chord $a_{k}$ of $\Lambda$; and $\partial$ is applied to matrices entry-by-entry. (The $\Delta_{l}$ matrices are diagonal with entries corresponding to the invertible generators $t_{l}^{i}$, which are associated to base points.) In passing from $\left(\mathcal{A}\left(\Lambda^{(k+1)}\right), \partial\right)$ to $\left(\mathcal{A}\left(\Lambda^{(k+1)}\right)^{\epsilon}, \partial^{\epsilon}\right)$, the right hand sides of the above formulas are adjusted by replacing the $t_{l}^{i}$ with $\epsilon\left(t_{l}^{i}\right)=\epsilon_{i}\left(t_{l}\right)$ and replacing all Reeb chord generators $s$ with $s+\epsilon(s)$. Since $\epsilon$ is pure, all $y_{l}^{i j}$ satisfy $\epsilon\left(y_{l}^{i j}\right)=0$, so for a generator $s^{1, k+1} \in F_{p} \mathcal{A}\left(\Lambda^{(k+1)}\right)^{\epsilon} \backslash F_{p-1} \mathcal{A}\left(\Lambda^{(k+1)}\right)^{\epsilon}$, the generators in $F_{p} \mathcal{A}\left(\Lambda^{(k+1)}\right)^{\epsilon} \backslash F_{p-1} \mathcal{A}\left(\Lambda^{(k+1)}\right)^{\epsilon}$ which appear in $\partial^{\epsilon} s^{1, k+1}$ must appear only in quadratic terms. Thus when $k \neq 2$ we can improve (4) to obtain $J=\max \left\{i_{1}, \ldots, i_{k}\right\}+1$, as desired.

Given objects $\epsilon, \epsilon^{\prime}$ in $\mathcal{A} u g_{+}(\Lambda ; \mathbb{k})$, we denote by $\operatorname{Hom}^{0}\left(\epsilon, \epsilon^{\prime}\right)^{\times}$the set of all elements $\alpha \in \operatorname{Hom}^{0}\left(\epsilon, \epsilon^{\prime}\right)$ such that $m_{1}(\alpha)=0$ and $\alpha$ has both right and left inverses with respect to the $m_{2}$ operations. As we have seen, the latter condition is equivalent to the coefficients of $y_{i}^{+}$in $\alpha$ belonging to $\mathbb{k}^{\times}$for $1 \leq i \leq \ell$, and given $m_{1}(\alpha)=0$ it implies that the left and right inverses of $\alpha$ are also cocycles. Thus $\operatorname{Hom}^{0}\left(\epsilon, \epsilon^{\prime}\right)^{\times}$is nonempty if and only if $\epsilon \cong \epsilon^{\prime}$.

The following corollary of Proposition 9 would easily be shown to hold in an honest category, but it requires extra care since $\mathcal{A} u g_{+}(\Lambda ; \mathbb{k})$ is an $A_{\infty}$ category and composition is not associative.

Proposition 10. If $\epsilon$ and $\epsilon^{\prime}$ are isomorphic objects of $\mathcal{A} u g_{+}\left(\Lambda ; \mathbb{k}_{k}\right)$, then there is a bijection $\operatorname{Hom}^{0}(\epsilon, \epsilon)^{\times} \cong \operatorname{Hom}^{0}\left(\epsilon, \epsilon^{\prime}\right)^{\times}$.

Proof. Fix $f \in \operatorname{Hom}^{0}\left(\epsilon, \epsilon^{\prime}\right)^{\times}$with left inverse $g \in \operatorname{Hom}^{0}\left(\epsilon^{\prime}, \epsilon\right)^{\times}$. The map $M_{f}: \operatorname{Hom}^{0}(\epsilon, \epsilon) \rightarrow \operatorname{Hom}^{0}\left(\epsilon, \epsilon^{\prime}\right)$, defined by $M_{f}(\beta)=m_{2}(f, \beta)$, and the similarly defined $M_{g}: \operatorname{Hom}^{0}\left(\epsilon, \epsilon^{\prime}\right) \rightarrow \operatorname{Hom}^{0}(\epsilon, \epsilon)$ are vector space isomorphisms. 
To see this, we compute using the $A_{\infty}$ relations that

$$
\begin{aligned}
M_{g} \circ M_{f}(\beta)= & m_{2}\left(g, m_{2}(f, \beta)\right) \\
= & m_{2}\left(m_{2}(g, f), \beta\right)+m_{1}\left(m_{3}(g, f, \beta)\right) \\
& \quad+m_{3}\left(m_{1}(g), f, \beta\right)+(-1)^{|g|} m_{3}\left(g, m_{1}(f), \beta\right)+(-1)^{|g|+|f|} m_{3}\left(g, f, m_{1}(\beta)\right) \\
= & \beta+m_{1}\left(m_{3}(g, f, \beta)\right)+m_{3}\left(g, f, m_{1}(\beta)\right) .
\end{aligned}
$$

Applying Proposition 9, we see that the matrix of $M_{g} \circ M_{f}$ with respect to the basis

$$
\left\{y_{1}, \ldots, y_{\ell}, a_{1}^{+}, \ldots, a_{r}^{+}\right\} \backslash\left\{a_{i}^{+}|| a_{i}^{+} \mid \neq 0\right\}
$$

has the form $I+N$ where $N$ is a strictly lower triangular matrix. Thus $M_{g} \circ M_{f}$ is injective, and in particular the map $M_{f}: \operatorname{Hom}^{0}(\epsilon, \epsilon) \rightarrow \operatorname{Hom}^{0}\left(\epsilon, \epsilon^{\prime}\right)$ is injective. By the same argument, we also see that $M_{g}: \operatorname{Hom}^{0}\left(\epsilon, \epsilon^{\prime}\right) \rightarrow$ $\operatorname{Hom}^{0}(\epsilon, \epsilon)$ is injective.

In addition, we claim that $M_{f}\left(\operatorname{Hom}^{0}(\epsilon, \epsilon)^{\times}\right) \subset \operatorname{Hom}^{0}\left(\epsilon, \epsilon^{\prime}\right)^{\times}$. Indeed, given $\alpha \in \operatorname{Hom}^{0}(\epsilon, \epsilon)^{\times}$, we have $m_{1}(\alpha)=$ 0 and $m_{1}(f)=0$ by definition, so

$$
m_{1}\left(M_{f}(\alpha)\right)=m_{2}\left(m_{1}(f), \alpha\right)+(-1)^{|f|} m_{2}\left(f, m_{1}(\alpha)\right)=0 .
$$

Also, the coefficients of the $y_{l}^{+}$in $m_{2}(f, \alpha)$ are products of the corresponding coefficients of $f$ and $\alpha$, and in particular are all units, so that $m_{2}(f, \alpha)$ has left and right inverses. This establishes that $M_{f}(\alpha) \in \operatorname{Hom}^{0}\left(\epsilon, \epsilon^{\prime}\right)^{\times}$. Similarly, $M_{g}$ sends $\operatorname{Hom}^{0}\left(\epsilon, \epsilon^{\prime}\right)^{\times}$to $\operatorname{Hom}^{0}(\epsilon, \epsilon)^{\times}$.

Since both $M_{f}$ and $M_{g}$ are injective as maps between $\operatorname{Hom}^{0}(\epsilon, \epsilon)^{\times}$and $\operatorname{Hom}^{0}\left(\epsilon, \epsilon^{\prime}\right)^{\times}$, it follows that $\operatorname{Hom}^{0}(\epsilon, \epsilon)^{\times}$ is in bijection with $\operatorname{Hom}^{0}\left(\epsilon, \epsilon^{\prime}\right)^{\times}$.

Remark. The same argument works to show that $\operatorname{Hom}^{0}(\epsilon, \epsilon)^{\times} \cong \operatorname{Hom}^{0}\left(\epsilon^{\prime}, \epsilon\right)^{\times}$, so repeated application of Proposition 10 shows that if $\epsilon_{0}, \epsilon_{1}, \epsilon_{0}^{\prime}, \epsilon_{1}^{\prime}: \mathcal{A}(\Lambda) \rightarrow \mathbb{k}$ are all isomorphic then there is a bijection $\operatorname{Hom}^{0}\left(\epsilon_{0}, \epsilon_{1}\right)^{\times} \cong$ $\operatorname{Hom}^{0}\left(\epsilon_{0}^{\prime}, \epsilon_{1}^{\prime}\right)^{\times}$.

Corollary 11. Fix $\epsilon: \mathcal{A}(\Lambda) \rightarrow \mathbb{F}_{q}$. Write $B^{0}(\epsilon, \epsilon)$ for the elements of $\operatorname{Hom}^{0}(\epsilon, \epsilon)$ which are coboundaries. Then

$$
\#\left\{\epsilon^{\prime}: \mathcal{A}(\Lambda) \rightarrow \mathbb{F}_{q} \mid \epsilon^{\prime} \cong \epsilon\right\}=q^{\operatorname{dim} \operatorname{Hom}^{0}(\epsilon, \epsilon)-\operatorname{dim} B^{0}(\epsilon, \epsilon)-\ell} \cdot \frac{(q-1)^{\ell}}{|\operatorname{Aut}(\epsilon)|} .
$$

Proof. Combining Corollary 8 and Proposition 10, we have shown that

$$
(q-1)^{\ell} q^{r^{\prime}}=\left|\bigsqcup_{\epsilon^{\prime} \cong \epsilon} \operatorname{Hom}^{0}\left(\epsilon, \epsilon^{\prime}\right)^{\times}\right|=\#\left\{\epsilon^{\prime} \mid \epsilon^{\prime} \cong \epsilon\right\} \cdot\left|\operatorname{Hom}^{0}(\epsilon, \epsilon)^{\times}\right| .
$$

But $\left|\operatorname{Hom}^{0}(\epsilon, \epsilon)^{\times}\right|$is $|\operatorname{Aut}(\epsilon)|$ times the number of coboundaries in $\operatorname{Hom}^{0}(\epsilon, \epsilon)$, which is $q^{\operatorname{dim} B^{0}(\epsilon, \epsilon)}$. The desired formula follows once we note in addition that $r^{\prime}=\operatorname{dim} \operatorname{Hom}^{0}(\epsilon, \epsilon)-\ell$.

We are now in a position to prove Theorem 1.

Proof of Theorem 1. Fix $\epsilon: \mathcal{A}(\Lambda) \rightarrow \mathbb{F}_{q}$. We expand the quantity $\operatorname{dim} \operatorname{Hom}^{0}(\epsilon, \epsilon)-\operatorname{dim} B^{0}(\epsilon, \epsilon)$. By definition, we have:

$$
\operatorname{dim} \operatorname{Hom}^{i}(\epsilon, \epsilon)=\operatorname{dim} B^{i}(\epsilon, \epsilon)+\operatorname{dim} H^{i} \operatorname{Hom}(\epsilon, \epsilon)+\operatorname{dim} B^{i+1}(\epsilon, \epsilon) .
$$

Summing over $i<0$,

$$
\sum_{i<0}(-1)^{i} \operatorname{dim} \operatorname{Hom}^{i}(\epsilon, \epsilon)=-\operatorname{dim} B^{0}(\epsilon, \epsilon)+\sum_{i<0}(-1)^{i} \operatorname{dim} H^{i} \operatorname{Hom}(\epsilon, \epsilon) .
$$

Note that we also have

$$
\chi_{*}=\sum_{i<0}(-1)^{i+1} r_{i}+\sum_{i \geq 0}(-1)^{i} r_{i}=-2 \ell+\sum_{i \leq 0}(-1)^{i} \operatorname{dim} \operatorname{Hom}^{i}(\epsilon, \epsilon)-\sum_{i>0}(-1)^{i} \operatorname{dim} \operatorname{Hom}^{i}(\epsilon, \epsilon)
$$

and

$$
-t b=\sum_{i}(-1)^{i} \operatorname{dim} \operatorname{Hom}^{i}(\epsilon, \epsilon)
$$


and hence

$$
\begin{aligned}
\frac{\chi_{*}-t b}{2} & =-\ell+\operatorname{dim} \operatorname{Hom}^{0}(\epsilon, \epsilon)+\sum_{i<0}(-1)^{i} \operatorname{dim} \operatorname{Hom}^{i}(\epsilon, \epsilon) \\
& =\operatorname{dim} \operatorname{Hom}^{0}(\epsilon, \epsilon)-\operatorname{dim} B^{0}(\epsilon, \epsilon)-\ell+\sum_{i<0}(-1)^{i} \operatorname{dim} H^{i} \operatorname{Hom}(\epsilon, \epsilon) .
\end{aligned}
$$

Thus we can rewrite the result of Corollary 11 as:

$$
\#\left\{\epsilon^{\prime}: \mathcal{A} \rightarrow \mathbb{F}_{q} \mid \epsilon^{\prime} \cong \epsilon\right\}=q^{\frac{\chi_{*}-t b}{2}} q^{-\sum_{i<0}(-1)^{i} \operatorname{dim} H^{i} \operatorname{Hom}(\epsilon, \epsilon)} \cdot \frac{(q-1)^{\ell}}{|\operatorname{Aut}(\epsilon)|} .
$$

Rearranging, we have

$$
\frac{\left|H^{-1} \operatorname{Hom}(\epsilon, \epsilon)\right| \cdot\left|H^{-3} \operatorname{Hom}(\epsilon, \epsilon)\right| \cdots}{|\operatorname{Aut}(\epsilon)| \cdot\left|H^{-2} \operatorname{Hom}(\epsilon, \epsilon)\right| \cdots}=q^{\frac{t b-\chi *}{2}} \cdot(q-1)^{-\ell} \cdot \#\left\{\epsilon^{\prime}: \mathcal{A} \rightarrow \mathbb{F}_{q} \mid \epsilon^{\prime} \cong \epsilon\right\},
$$

and summing over the isomorphism classes on both sides gives the assertion of Theorem 1.

\section{Doubly LAGRANGiAn SLICE KNOTS}

Given Legendrian knots $\Lambda_{-}, \Lambda_{+} \subset \mathbb{R}^{3}$, we say that $\Lambda_{-}$is Lagrangian concordant to $\Lambda_{+}$, denoted $\Lambda_{-} \prec \Lambda_{+}$, if there is a Lagrangian cylinder $L$ in the symplectization $\left(\mathbb{R} \times \mathbb{R}^{3}, d\left(e^{t}(d z-y d x)\right)\right)$ and some $T \gg 0$ such that

$$
L \cap\left(\{t\} \times \mathbb{R}^{3}\right)= \begin{cases}\{t\} \times \Lambda_{-}, & t \leq-T \\ \{t\} \times \Lambda_{+}, & t \geq T\end{cases}
$$

The Lagrangian $L$ in this case is called a Lagrangian concordance from $\Lambda_{-}$to $\Lambda_{+}$. If $U$ is the standard Legendrian unknot with $t b(U)=-1$, and we have both $U \prec \Lambda$ and $\Lambda \prec U$, we will say that $\Lambda$ is doubly Lagrangian slice. In this section we will use Corollary 2 to understand $\mathcal{A} u g_{+}(\Lambda)$ for such $\Lambda$.

Theorem 12 ([CNS]). If $\Lambda$ is doubly Lagrangian slice, then its ruling polynomial ${ }^{2}$ satisfies $R_{\Lambda}(z)=z^{-1}$.

A doubly Lagrangian slice knot must have $t b(\Lambda)=-1$, since the Thurston-Bennequin number is invariant under Lagrangian concordance [Cha10], so according to Corollary 2 such knots satisfy

$$
\# \pi_{\geq 0} \mathcal{A} u g_{+}\left(\Lambda ; \mathbb{F}_{q}\right)^{*}=q^{-1 / 2}\left(q^{1 / 2}-q^{-1 / 2}\right)^{-1}=\frac{1}{q-1} .
$$

Now given a Lagrangian concordance $L$ from $\Lambda_{-}$to $\Lambda_{+}$, the work of $[\mathrm{EHK}]$ produces a dga morphism

$$
\Phi_{L}: \mathcal{A}\left(\Lambda_{+}\right) \rightarrow \mathcal{A}\left(\Lambda_{-}\right)
$$

between the dgas of $\Lambda_{ \pm}$. In [EHK], these dgas are considered over $\mathbb{Z} / 2$, but in fact it is known that the result still holds for the dgas over $\mathbb{Z}\left[t, t^{-1}\right]$ as in [ENS02]. More precisely, the cobordism map $\Phi_{L}$ as presented in [EHK] counts gradient flow trees for a Morse cobordism, and these trees in turn correspond to holomorphic disks for Legendrian contact homology in $J^{1}([0,1] \times \mathbb{R})$, the 1 -jet space of a strip, by [Ekh07]. Orientations for the moduli spaces of these disks have been worked out in [EES05], and it follows that we can lift $\Phi_{L}$ from a map over $\mathbb{Z} / 2$ to a map over $\mathbb{Z}$. To lift this further to $\mathbb{Z}\left[t, t^{-1}\right]$, choose base points on $\Lambda_{ \pm}$and connect them by a path $\gamma$ in $L$; then the powers of $t$ in the lifted map $\Phi_{L}$ count signed intersections of the flow trees with $\gamma$.

By $\left[\mathrm{NRS}^{+} 15\right.$, Proposition 3.29], the map $\Phi_{L}$ of dgas over $\mathbb{Z}\left[t, t^{-1}\right]$ induces a functor

$$
F_{L}: \mathcal{A} u g_{+}\left(U ; \mathbb{F}_{q}\right) \rightarrow \mathcal{A} u g_{+}\left(\Lambda ; \mathbb{F}_{q}\right)
$$

of unital $A_{\infty}$ categories. Let $\epsilon_{U}$ be the unique augmentation in $\mathcal{A} u g_{+}\left(U ; \mathbb{F}_{q}\right)$, and write $\epsilon_{L}$ for the augmentation of $\mathcal{A}(\Lambda)$ that is the image of $\epsilon_{U}$ under $F_{L}$; note that $\epsilon_{L}=\epsilon_{U} \circ \Phi_{L}$ and $\epsilon_{L}$ is the augmentation induced by capping off the concave end of $L$ to get a Lagrangian disk filling of $\Lambda$.

Proposition 13. If $\Lambda$ is doubly Lagrangian slice, then all augmentations $\epsilon: \mathcal{A}(\Lambda) \rightarrow \mathbb{F}_{q}$ are homotopic to $\epsilon_{L}$. [CNS].

${ }^{2}$ In [CNS] the stated theorem is $R_{\Lambda}(z)=1$, but we use the normalization $R_{\Lambda}(z)=\sum_{R \in \mathcal{R}} z^{-\chi(R)}$ rather than the sum $\sum z^{1-\chi(R)}$ of 
Proof. Since $\epsilon_{L}$ is induced by a disk filling of $\Lambda$, [NRS ${ }^{+} 15$, Proposition 5.7] says that $H^{*} \operatorname{Hom}\left(\epsilon_{L}, \epsilon_{L}\right) \cong H^{*}\left(D^{2} ; \mathbb{F}_{q}\right)$. In particular, the cohomology of $\operatorname{Hom}\left(\epsilon_{L}, \epsilon_{L}\right)$ is supported in degree zero and has rank 1, so the only possible automorphisms of $\epsilon_{L}$ are nonzero scalar multiples of the unit $e_{\epsilon_{L}}$ and there are no higher homotopies. The isomorphism class of $\epsilon_{L}$ therefore contributes $\frac{1}{\left|\mathbb{F}_{q}^{\times}\right|}=\frac{1}{q-1}$ to the homotopy cardinality of $\mathcal{A} u g_{+}\left(\Lambda ; \mathbb{F}_{q}\right)^{*}$. But we have seen above that this is the entire homotopy cardinality, so all augmentations of $\Lambda$ are isomorphic to $\epsilon_{L}$. It follows by [NRS ${ }^{+} 15$, Proposition 5.17] that all augmentations of $\Lambda$ are homotopic to $\epsilon_{L}$.

Remark. In fact, this can be strengthened somewhat, using additional results from [CNS]: if $U \prec \Lambda$ and $\Lambda \prec U$ then the Legendrian $n$-stranded cable $S\left(\Lambda, t w_{n}\right)$ (see [CNS, Section 2.4]), which is topologically the Seifert-framed $n$-cable of $\Lambda$, has ruling polynomial $z^{-n}$ and $t b=-n$, hence $\# \pi_{\geq 0} \mathcal{A} u g_{+}\left(S\left(\Lambda, t w_{n}\right) ; \mathbb{F}_{q}\right)^{*}=\frac{1}{(q-1)^{n}}$. Given a Lagrangian concordance $L$ from $U$ to $\Lambda$, we produce a Lagrangian concordance $L^{n}$ (i.e. a disjoint union of $n$ Lagrangian cylinders) from $S\left(U, t w_{n}\right)$ to $S\left(\Lambda, t w_{n}\right)$, and since $S\left(U, t w_{n}\right)$ is Legendrian isotopic to $n$ unlinked copies of $U$, it admits a filling by $n$ Lagrangian disks $\bigsqcup_{i=1}^{n} D^{2}$. Let $\epsilon_{U}$ be the induced augmentation; then $\epsilon_{L^{n}}=F_{L^{n}}\left(\epsilon_{U}\right)$ corresponds to the filling $\left(\sqcup D^{2}\right) \cup_{S\left(\Lambda, t w_{n}\right)} L^{n}$ of $S\left(\Lambda, t w_{n}\right)$. It follows that

$$
H^{*} \operatorname{Hom}\left(\epsilon_{U}, \epsilon_{U}\right) \cong H^{*} \operatorname{Hom}\left(\epsilon_{L^{n}}, \epsilon_{L^{n}}\right)=H^{*}\left(D^{2} \sqcup \cdots \sqcup D^{2} ; \mathbb{F}_{q}\right) \cong \mathbb{F}_{q}^{\oplus n}
$$

as abelian groups, supported in degree 0 . If we can show that $H^{*} \operatorname{Hom}\left(\epsilon_{L^{n}}, \epsilon_{L^{n}}\right) \cong \mathbb{F}_{q}^{\oplus n}$ as a ring, then it will follow that $\left|\operatorname{Aut}\left(\epsilon_{L^{n}}\right)\right|=(q-1)^{n}$, and hence that all augmentations of $S\left(\Lambda, t w_{n}\right)$ are homotopic to $\epsilon_{L^{n}}$ just as in the proof of Proposition 13. This is a special case of the statement that the isomorphism $H^{*} \operatorname{Hom}\left(\epsilon_{L}, \epsilon_{L}\right) \cong H^{*}(L)$ is a ring isomorphism for any exact Lagrangian filling $L$, which should be provable by considering $Y$-shaped gradient flow trees on $L$; here we prove just the special case that we need.

If $L^{\prime}$ is a Lagrangian concordance from $\Lambda$ to $U$ and $\left(L^{\prime}\right)^{n}$ is the corresponding cable of $L^{\prime}$, then $L \cup_{\Lambda} L^{\prime}$ is Hamiltonian isotopic to a product concordance [EP96, Cha13], hence so is $L^{n} \cup_{S\left(\Lambda, t w_{n}\right)}\left(L^{\prime}\right)^{n}$. The ring homomorphism

$$
F_{\left(L^{\prime}\right)^{n}} \circ F_{L^{n}}=F_{L^{n} \cup_{S\left(\Lambda, t w_{n}\right)}\left(L^{\prime}\right)^{n}}: H^{*} \operatorname{Hom}\left(\epsilon_{U}, \epsilon_{U}\right) \rightarrow H^{*} \operatorname{Hom}\left(\epsilon_{U}, \epsilon_{U}\right)
$$

is therefore an isomorphism, and so $F_{L^{n}}: H^{*} \operatorname{Hom}\left(\epsilon_{U}, \epsilon_{U}\right) \rightarrow H^{*} \operatorname{Hom}\left(\epsilon_{L^{n}}, \epsilon_{L^{n}}\right)$ is injective. Both of these rings have cardinality $q^{n}$, so the map is bijective and hence an isomorphism of rings; and a direct computation shows that $H^{*} \operatorname{Hom}\left(\epsilon_{U}, \epsilon_{U}\right)=\mathbb{F}_{q}^{\oplus n}$ as rings, so the same is true of $H^{*} \operatorname{Hom}\left(\epsilon_{L^{n}}, \epsilon_{L^{n}}\right)$ as claimed.

By Proposition 13, if $\Lambda$ is doubly Lagrangian slice, then $F_{L}: \mathcal{A} u g_{+}\left(U ; \mathbb{F}_{q}\right) \rightarrow \mathcal{A} u g_{+}\left(\Lambda ; \mathbb{F}_{q}\right)$ maps between categories that each have one object up to equivalence. In fact, we can say more. Say that an $A_{\infty}$ functor between unital $A_{\infty}$ categories is an $A_{\infty}$ quasi-equivalence if the induced functor on cohomology categories is fully faithful and essentially surjective.

Theorem 14. If $\Lambda$ is doubly Lagrangian slice and $L$ is a Lagrangian concordance from $U$ to $\Lambda$, then the induced functor $F_{L}: \mathcal{A} u g_{+}\left(U ; \mathbb{F}_{q}\right) \rightarrow \mathcal{A} u g_{+}\left(\Lambda ; \mathbb{F}_{q}\right)$ is an $A_{\infty}$ quasi-equivalence.

Proof. We revisit the proof of Proposition 13. Since all objects of $\mathcal{A} u g_{+}(\Lambda)$ are isomorphic to $\epsilon_{L}$, the functor $F_{L}$ is essentially surjective. Moreover, the induced map $H^{*} \operatorname{Hom}\left(\epsilon_{U}, \epsilon_{U}\right) \rightarrow H^{*} \operatorname{Hom}\left(\epsilon_{L}, \epsilon_{L}\right)$ is an isomorphism, since $F_{L}\left(e_{\epsilon_{U}}\right)=e_{\epsilon_{L}}$ and the cohomology rings are spanned by the classes $\left[e_{\epsilon_{U}}\right]$ and $\left[e_{\epsilon_{L}}\right]$ respectively, so $F_{L}$ is also fully faithful on cohomology.

In light of Theorem 14, a natural question to ask is whether the underlying dga map $\Phi_{L}$ is a quasi-isomorphism (or, better yet, a stable tame isomorphism, which would automatically imply Theorem 14). This is currently an open question, but Theorem 14 does imply that the map $\Phi_{L}$ is a quasi-isomorphism on a certain completion of the dgas.

Given an augmentation $\epsilon: \mathcal{A}(\Lambda) \rightarrow \mathbb{F}_{q}$, we follow the notation of [NRS $\left.{ }^{+} 15\right]$ : let $\mathcal{A}^{\epsilon}=\left(\mathcal{A} \otimes \mathbb{F}_{q}\right) /(t=\epsilon(t))$ (this is the unital tensor algebra over $\mathbb{F}_{q}$ generated by Reeb chords of $\Lambda$, and the differential $\partial$ descends to $\left.\mathcal{A}^{\epsilon}\right)$, and let $\mathcal{A}_{+}^{\epsilon}$ denote the subspace of $\mathcal{A}^{\epsilon}$ generated by all nonempty words of Reeb chords. Define $\phi_{\epsilon}: \mathcal{A}^{\epsilon} \rightarrow \mathcal{A}^{\epsilon}$ to be the algebra automorphism determined by $\phi_{\epsilon}(a)=a+\epsilon(a)$ for all Reeb chords $a$. Then the differential $\partial_{\epsilon}:=\phi_{\epsilon} \circ \partial \circ \phi_{\epsilon}^{-1}$ on $\mathcal{A}^{\epsilon}$ is filtered with respect to the filtration

$$
\mathcal{A}^{\epsilon} \supset \mathcal{A}_{+}^{\epsilon} \supset\left(\mathcal{A}_{+}^{\epsilon}\right)^{2} \supset \cdots .
$$

Let $\widehat{\mathcal{A}}^{\epsilon}(\Lambda)$ denote the completion of $\mathcal{A}^{\epsilon}$ with respect to this filtration, and note that $\partial_{\epsilon}$ extends to a differential on $\widehat{\mathcal{A}}^{\epsilon}(\Lambda)$.

Proposition 15. If $\Lambda$ is doubly Lagrangian slice and $L$ is a Lagrangian concordance from $U$ to $\Lambda$, then the morphism

$$
\Phi_{L}: \widehat{\mathcal{A}}^{\epsilon_{L}}(\Lambda) \rightarrow \widehat{\mathcal{A}}^{\epsilon_{U}}(U)
$$


is a quasi-isomorphism.

Proof. When $(\mathcal{A}, \partial)$ is the dga for a Legendrian knot and $\epsilon: \mathcal{A} \rightarrow \mathbb{F}_{q}$ is an augmentation, the twisted dga $\left(\mathcal{A}^{\epsilon}, \partial_{\epsilon}\right)$ dualizes to the $A_{\infty}$ algebra Hom_ $(\epsilon, \epsilon)$, where Hom - denotes morphism in the Bourgeois-Chantraine augmentation category $\mathcal{A u g}_{-}\left(\Lambda ; \mathbb{F}_{q}\right)\left[\mathrm{BC} 14, \mathrm{NRS}^{+} 15\right]$. Note that Hom $-(\epsilon, \epsilon)$ is a subcomplex of $\mathrm{Hom}_{+}(\epsilon, \epsilon)$, and that in our setting the $A_{\infty}$ functor $F_{L}: \mathcal{A} u g_{+}\left(U ; \mathbb{F}_{q}\right) \rightarrow \mathcal{A} u g_{+}\left(\Lambda ; \mathbb{F}_{q}\right)$ in particular gives chain maps $F_{L} \operatorname{Hom}_{ \pm}\left(\epsilon_{U}, \epsilon_{U}\right) \rightarrow$ $\operatorname{Hom}_{ \pm}\left(\epsilon_{L}, \epsilon_{L}\right)$. We then have the following commutative diagram:

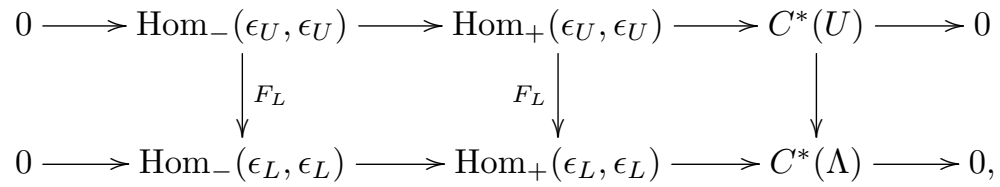

where the exact rows are as in $\left[\mathrm{NRS}^{+} 15\right.$, Proposition 5.2].

By Theorem 14, the middle column induces an isomorphism on cohomology, and so the left column does as well. That is, $F_{L}: \mathcal{A} u g_{-}\left(U ; \mathbb{F}_{q}\right) \rightarrow \mathcal{A} u g_{-}\left(\Lambda ; \mathbb{F}_{q}\right)$ is an $A_{\infty}$ quasi-equivalence. The result now follows by noting that the duals of the $A_{\infty}$ algebras $\mathcal{A} u g_{-}\left(U ; \mathbb{F}_{q}\right), \mathcal{A} u g_{-}\left(\Lambda ; \mathbb{F}_{q}\right)$ are the completed dgas $\left(\widehat{\mathcal{A}}^{\epsilon_{U}}(U), \partial_{\epsilon_{U}}\right)$ and $\left(\widehat{\mathcal{A}}^{\epsilon_{L}}(\Lambda), \partial_{\epsilon_{L}}\right)$, and that the dual map to $F_{L}: \mathcal{A} u g_{-}\left(U ; \mathbb{F}_{q}\right) \rightarrow \mathcal{A} u g_{-}\left(\Lambda ; \mathbb{F}_{q}\right)$ is $\Phi_{L}$.

Remark. Although we would like to replace the completions $\widehat{\mathcal{A}}$ by the original algebras $\mathcal{A}$ in the statement of Proposition 15 , our technique does not allow us to do this directly. Indeed, it is possible for two (augmented) dgas $(\mathcal{A}, \partial)$ and $\left(\mathcal{A}^{\prime}, \partial^{\prime}\right)$ to have dual $A_{\infty}$ algebras that are $A_{\infty}$ quasi-isomorphic without the original dgas being quasi-isomorphic. Let $\mathbb{k}$ be a field, let $\mathcal{A}=\mathbb{k}$ with $\partial=0$, and let $\mathcal{A}^{\prime}$ be the tensor algebra over $\mathbb{k}$ generated by $a, b$ with $\partial^{\prime}(a)=b-b^{2}$, $\partial^{\prime}(b)=0,|a|=1,|b|=0$. The $A_{\infty}$ algebra dual to $\left(\mathcal{A}^{\prime}, \partial^{\prime}\right)$ has generators $a^{*}, b^{*}$ with $m_{1}\left(b^{*}\right)=a^{*}, m_{1}\left(a^{*}\right)=0$, and thus the $m_{1}$ cohomologies of the $A_{\infty}$ algebras dual to both $(\mathcal{A}, \partial)$ and $\left(\mathcal{A}^{\prime}, \partial^{\prime}\right)$ are 0 . The inclusion map $i: \mathcal{A} \rightarrow \mathcal{A}^{\prime}$ thus induces an isomorphism on cohomology on the dual $A_{\infty}$ algebras. However, $H_{0}(\mathcal{A}, \partial)=\mathbb{k}$, while $H_{0}\left(\mathcal{A}^{\prime}, \partial^{\prime}\right) \cong \mathbb{k}[b] /\left(b-b^{2}\right)$, and so $i_{*}: H(\mathcal{A}, \partial) \rightarrow H\left(\mathcal{A}^{\prime}, \partial^{\prime}\right)$ is not an isomorphism in this case.

\section{Counting $2 m$-GRAded Augmentations}

So far, we have restricted ourselves to the setting where the dga has a $\mathbb{Z}$ grading and augmentations and rulings respect this grading. In this section, we sketch how to extend our results on counting augmentations to the more general setting of a $\mathbb{Z} /(2 m)$ grading for some integer $m$. By a result of Sabloff [Sab05, Theorem 1.3], $\Lambda$ can have $2 m$-graded augmentations and rulings only if the rotation number $r(\Lambda)$ is 0 . In this section, we further assume that each component of $\Lambda$ has rotation number 0 ; this is no additional condition if $\Lambda$ is connected, but is necessary to define the shifted Euler characteristic $\chi_{*}$ below. In addition, if $\Lambda$ has multiple components, we equip each component of $\Lambda$ with a Maslov potential, in order to define the degrees of Reeb chords between different components of $\Lambda$ and thus the grading on the augmentation category; in fact, this is implicitly done in the $\mathbb{Z}$-graded setting as well.

Let $m \geq 0$, and fix a finite field $\mathbb{F}_{q}$ as before. As described in [NRS $\left.{ }^{+} 15\right]$, we can construct the $\mathbb{Z} /(2 m)$-graded augmentation category of $\Lambda$, which we again write as $\mathcal{A} u g_{+}\left(\Lambda ; \mathbb{F}_{q}\right)$, whose objects are $2 m$-graded augmentations (i.e., $\epsilon: \mathcal{A}(\Lambda) \rightarrow \mathbb{F}_{q}$ with $\epsilon(a)=0$ unless $\left.|a| \equiv 0(\bmod 2 m)\right)$, and whose morphisms are $\mathbb{Z} /(2 m)$-graded vector spaces over $\mathbb{F}_{q}$. We write $\mathcal{R}$ for the set of $2 m$-graded normal rulings of $\Lambda$, and define the $2 m$-graded ruling polynomial $R_{\Lambda}^{2 m}(z)=\sum_{R \in \mathcal{R}} z^{-\chi(R)}$. When $m=0$, this recovers the definitions from before. A case of particular interest is $m=1$, where $z R_{\Lambda}^{2}(z)$ is the coefficient of $a^{-t b(\Lambda)-1}$ in the HOMFLY-PT polynomial $P_{\Lambda}(a, z)$, as shown in [Rut06].

Since the naive number of augmentations changes under stabilization of the dga, we need a normalized augmentation count that can then be related to the ruling polynomial as in Equation (1) from the Introduction. For $m=0$, the normalization is given by $q^{-\chi_{*}(\Lambda) / 2} \#\left\{\mathcal{A}(\Lambda) \rightarrow \mathbb{F}_{q}\right\}$ where $\chi_{*}$ is the shifted Euler characteristic as defined in the Introduction; a similar expression is given in [NS06] in the odd-graded case. The key in the $\mathbb{Z}$-graded case $(m=0)$ is that if we stabilize $\mathcal{A}$ by adding two generators of degrees $i$ and $i-1$, then $\#\left\{\mathcal{A} \rightarrow \mathbb{F}_{q}\right\}$ is multiplied by $q$ when $i=0$ and is unchanged otherwise, while $\chi_{*}(\Lambda)$ increases by 2 when $i=0$ and is unchanged otherwise.

Now let $m>0$; to obtain a similar result in the $\mathbb{Z} /(2 m)$-graded case, we modify the definition of $\chi_{*}$ as follows. Since each component of $\Lambda$ has rotation number 0 by assumption, $\mathcal{A}(\Lambda)$ is $\mathbb{Z}$-graded, and we can set $r_{i}$ to be the number of Reeb chords of degree $i$ as before. Now let

$$
s_{k}=r_{2 m k}-r_{2 m k+1}+\cdots+r_{2 m k+2 m-2}-r_{2 m k+2 m-1},
$$


and define

$$
\chi_{*}(\Lambda)=\sum_{k}(2 k+1) s_{k} .
$$

As in the $m=0$ case, this has the property that stabilizing $\mathcal{A}$ by adding generators of degree $i$ and $i-1$ increases $\chi_{*}$ by 2 if $i \equiv 0(\bmod 2 m)$ and leaves $\chi_{*}$ unchanged otherwise. Since $\#\left\{\mathcal{A}(\Lambda) \rightarrow \mathbb{F}_{q}\right\}$, the number of $2 m$ graded augmentations $\mathcal{A} \rightarrow \mathbb{F}_{q}$, is multiplied by $q$ in the first case and is unchanged in the second, it follows that $q^{-\chi_{*}(\Lambda) / 2} \#\left\{\mathcal{A}(\Lambda) \rightarrow \mathbb{F}_{q}\right\}$ is invariant under stable tame isomorphism.

A result from [HR15] together with a nontrivial extension of Lemma 5 from [NS06] (the extension is stated here as Lemma 17, and we limit ourselves to a sketch of the proof) can be used to show the following.

Proposition 16. For any $m \geq 0$, we have

$$
q^{-\chi_{*}(\Lambda) / 2}(q-1)^{-\ell} \cdot \#\left\{\mathcal{A}(\Lambda) \rightarrow \mathbb{F}_{q}\right\}=R_{\Lambda}^{2 m}\left(q^{1 / 2}-q^{-1 / 2}\right) .
$$

Note that for $m=0$, this is Equation (1) from the Introduction.

Proof. Given a $2 m$-graded normal ruling $R$ of $\Lambda$, those crossings with degree $0(\bmod 2 m)$ are divided into three classes: departures, returns, and switches. At a departure (resp. return) $c$, the two disks whose boundaries meet at $c$ satisfy the normality condition, i.e., they are disjoint or nested, at values of $x$ immediately preceding (resp. following) c. Now [HR15, Theorem 3.4] shows that (under the assumption that $\Lambda$ is the resolution of a plat position front diagram, which can always be made after a Legendrian isotopy) the number of $2 m$-graded augmentations is

$$
\#\left\{\mathcal{A}(\Lambda) \rightarrow \mathbb{F}_{q}\right\}=\sum_{R \in \mathcal{R}}(q-1)^{-\chi(R)+\ell} \cdot q^{\# \text { returns }} .
$$

The equality (5) then follows in a straightforward manner from the following generalization of [NS06, Lemma 5].

Lemma 17. Suppose $\Lambda$ is the resolution of a front diagram in plat position. For any $2 m$-graded ruling $R$ of $\Lambda$, we have

$$
\text { \#returns }=\frac{1}{2}\left(\chi_{*}(\Lambda)+\chi(R)\right) .
$$

Sketch of proof. For $k \in \mathbb{Z}$, let $C_{k}$ denote the number of crossings on the front projection of $\Lambda$ with degree $k$, and when $k \equiv 0(\bmod 2) m$, write

$$
C_{k}=D_{k}+R_{k}+S_{k}
$$

where $D_{k}, R_{k}$, and $S_{k}$ respectively denote the number of departures, returns, and switches of degree $k$. Since $\Lambda$ is the resolution of a front diagram, the Reeb chords of $\Lambda$ are in one-to-one correspondence with crossings and right cusps of the front of $\Lambda$, and so

$$
r_{k}= \begin{cases}C_{k} & k \neq 1 \\ C_{k}+\# \text { right cusps } & k=1\end{cases}
$$

along with the fact that $\chi(R)=\#$ right cusps - \#switches, this implies that (6) is equivalent to:

(7) $0=\sum_{k \equiv 0(\bmod 2 m)}\left(\left(\frac{k}{m}+1\right) D_{k}+\left(\frac{k}{m}-1\right) R_{k}+\left(\frac{k}{m}\right) S_{k}\right)+\sum_{k \neq 0(\bmod 2 m)}(-1)^{k}\left(2\left\lfloor\frac{k}{2 m}\right\rfloor+1\right) C_{k}$.

To establish (7), we associate an integer $I(x)$ to values of $x \in \mathbb{R}$ that are not the $x$ coordinates of crossings or cusps of the front projection of $\Lambda$. The function $I$ will have the properties:

(i) As $x$ moves from left to right in $\mathbb{R}, I(x)$ is constant except when $x$ passes a crossing of $\Lambda$.

(ii) $I(x)=0$ for $x \ll 0$ and $x \gg 0$.

(iii) Suppose that a single crossing $c$ occurs in the interval $\left(x_{0}, x_{1}\right)$. Then:

$$
I\left(x_{1}\right)-I\left(x_{0}\right)= \begin{cases}\frac{|c|}{m}+1 & \text { if } c \text { is a departure, } \\ \frac{|c|}{m}-1 & \text { if } c \text { is a return, } \\ \frac{|c|}{m} & \text { if } c \text { is a switch, } \\ (-1)^{|c|}\left(2\left\lfloor\frac{|c|}{2 m}\right\rfloor+1\right) & \text { if }|c| \not \equiv 0 \quad(\bmod 2 m) .\end{cases}
$$


The equation (7) then follows from considering the total change in $I$ from $x \ll 0$ to $x \gg 0$.

To define $I$ we introduce some notation. Let $D_{1}, \ldots, D_{n}$ denote the disks that constitute the ruling $R$. Each $D_{i}$ has an upper strand and a lower strand; we denote the value of the (ZZZ-valued) Maslov potential on the upper (resp. lower) strand of $D_{i}$ by $a_{i}$ (resp. $b_{i}$ ). Note that these values may change along the upper and lower strands of $D_{i}$ but only by multiples of $2 m$.

We now define

$$
I(x)=I_{1}(x)+I_{2}(x)+I_{3}(x),
$$

where $I_{1}, I_{2}, I_{3}$ are given as follows. The first term is defined by

$$
\begin{aligned}
I_{1}(x) & =\sum_{a_{i}} \sum_{b_{i}}(-1)^{b_{i}-a_{j}}\left(2\left\lfloor\frac{b_{i}-a_{j}}{2 m}\right\rfloor+1\right) \\
a_{j} & b_{j}
\end{aligned}
$$

where the sum is over all pairs of interlaced disks at $x, D_{i}$ and $D_{j}$, with $D_{i}$ the upper of the two disks. Next,

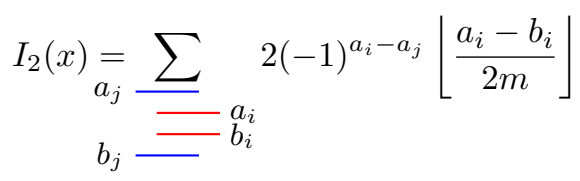

where the sum is over all pairs of nested disks, $E_{i}$ and $E_{j}$, with $E_{i}$ inside $E_{j}$ at $x$. Finally,

$$
I_{3}(x)=\underset{a_{i}}{=} \sum\left\lfloor\frac{a_{i}-b_{i}}{2 m}\right\rfloor
$$

where the sum is over all disks at $x$.

That the properties (i) and (ii) hold is easily verified. That $I(x)$ satisfies (iii) can be verified by a lengthy but fairly straightforward check based on considering the combinatorics of the ruling disks that meet at a crossing $c$ in a case-bycase manner. When $c$ is a switch, one also needs to consider the possible ways in which a third disk $D_{k}$ may intersect $D_{i}$ and $D_{j}$.

Proposition 16 relates the naive count of augmentations $\#\left\{\mathcal{A}(\Lambda) \rightarrow \mathbb{F}_{q}\right\}$ to the $2 m$-graded ruling polynomial. On the other hand, the same argument as in Section 2, but now in the $2 m$-graded setting, yields the following:

$$
\#\left\{\mathcal{A}(\Lambda) \rightarrow \mathbb{F}_{q}\right\}=\sum_{[\epsilon]} \frac{(q-1)^{\ell}}{|\operatorname{Aut}(\epsilon)|} q^{\operatorname{dim} \operatorname{Hom}^{0}(\epsilon, \epsilon)-\operatorname{dim} B^{0}(\epsilon, \epsilon)-\ell} .
$$

Here we note that all gradings are taken in $\mathbb{Z} /(2 m)$, and that the sum is over isomorphism classes of augmentations in the $2 m$-graded category. Note in particular that the exponent $\operatorname{dim} \operatorname{Hom}^{0}(\epsilon, \epsilon)-\operatorname{dim} B^{0}(\epsilon, \epsilon)-\ell$ in the summand is independent of the choice of representative $\epsilon$ of the isomorphism class $[\epsilon]$, cf. Corollary 11.

Combined with Proposition 16, this yields the following.

Proposition 18. For any $m \geq 0$, we have

$$
\sum_{[\epsilon]} \frac{1}{|\operatorname{Aut}(\epsilon)|} q^{\operatorname{dim} \operatorname{Hom}^{0}(\epsilon, \epsilon)-\operatorname{dim} B^{0}(\epsilon, \epsilon)-\ell-\left(\chi_{*}(\Lambda)-t b(\Lambda)\right) / 2}=q^{t b(\Lambda) / 2} R_{\Lambda}\left(q^{1 / 2}-q^{-1 / 2}\right) .
$$

By comparison with Corollary 2, it then makes sense to regard the left hand side of the equation in Proposition 18 as being the homotopy cardinality of the $2 m$-graded augmentation category for $m \geq 0$. Indeed, for $m=0$, the proof of Theorem 1 shows that

$$
q^{\operatorname{dim} \operatorname{Hom}^{0}(\epsilon, \epsilon)-\operatorname{dim} B^{0}(\epsilon, \epsilon)-\ell-\left(\chi_{*}(\Lambda)-t b(\Lambda)\right) / 2}=\frac{\left|H^{-1} \operatorname{Hom}(\epsilon, \epsilon)\right| \cdot\left|H^{-3} \operatorname{Hom}(\epsilon, \epsilon)\right| \cdots}{\left|H^{-2} \operatorname{Hom}(\epsilon, \epsilon)\right| \cdot\left|H^{-4} \operatorname{Hom}(\epsilon, \epsilon)\right| \cdots}
$$

and so the left hand side is indeed $\pi_{\geq 0} \mathcal{A} u g_{+}\left(\Lambda ; \mathbb{F}_{q}\right)^{*}$.

For $m>0$, there are two deficiencies with viewing the left hand side in Proposition 18 as the homotopy cardinality: first, there is no obvious relation to the notion of homotopy cardinality from homotopy theory; and second, the expression involves ranks of chain and boundary groups, rather than cohomology groups, which are more natural from the viewpoint of proving invariance directly. We do not address the first problem here, but propose a conjectural way to resolve the second. There is a fair amount of experimental evidence for the following. 
Conjecture 19. Let $\epsilon: \mathcal{A}(\Lambda) \rightarrow \mathbb{F}_{q}$ be a $2 m$-graded augmentation of a Legendrian link $\Lambda$ with $\ell$ components, for $m \geq 0$. Then

$$
2 \operatorname{dim} \operatorname{Hom}^{0}(\epsilon, \epsilon)-2 \operatorname{dim} B^{0}(\epsilon, \epsilon)-\ell-\chi_{*}(\Lambda)=2 \operatorname{dim} H^{0} \operatorname{Hom}(\epsilon, \epsilon)-\operatorname{dim} H^{1} \operatorname{Hom}(\epsilon, \epsilon) .
$$

Conjecture 19, combined with Proposition 18, would have the following consequence.

Corollary 20. For any $m \geq 0$,

$$
\sum_{[\epsilon]} \frac{1}{|\operatorname{Aut}(\epsilon)|} \frac{\left|H^{0} \operatorname{Hom}(\epsilon, \epsilon)\right|}{\left|H^{1} \operatorname{Hom}(\epsilon, \epsilon)\right|^{1 / 2}} q^{(t b(\Lambda)-\ell) / 2}=q^{t b(\Lambda) / 2} \sum_{R \in \mathcal{R}}\left(q^{1 / 2}-q^{-1 / 2}\right)^{-\chi(R)} .
$$

It would then be reasonable to define the left hand side of Corollary 20 as the homotopy cardinality of the $2 m$-graded augmentation category.

Remark. In the case $m=0$, the left hand side agrees with the definition of $\pi_{>0} \mathcal{A} u g_{+}\left(\Lambda ; \mathbb{F}_{q}\right)^{*}$ from the Introduction, as can be seen directly by Sabloff duality (see the proof of Proposition 21 below). For $m=1$, since $t b(\Lambda)=$ $\operatorname{dim} H^{1} \operatorname{Hom}(\epsilon, \epsilon)-\operatorname{dim} H^{0} \operatorname{Hom}(\epsilon, \epsilon)$, the left hand side of Corollary 20 is $\sum_{[\epsilon]} \frac{1}{|\operatorname{Aut}(\epsilon)|}\left|H^{0} \operatorname{Hom}(\epsilon, \epsilon)\right|^{1 / 2} q^{-\ell / 2}$.

We conclude this section by establishing Conjecture 19 in a case that generalizes $m=0$. Suppose that $m$ is arbitrary but the augmentation $\epsilon$ is not just $\mathbb{Z} /(2 m)$-graded but in fact $\mathbb{Z}$-graded, i.e., $\epsilon(a)=0$ unless $|a|=0$. Then the space $\operatorname{Hom}(\epsilon, \epsilon)$ has a $\mathbb{Z}$ grading that descends to a $\mathbb{Z} /(2 m)$ grading.

Proposition 21. Suppose that $\epsilon$ is $\mathbb{Z}$-graded and thus $\mathbb{Z} /(2 m)$-graded for any $m \geq 0$. Then Conjecture 19 holds for any $m \geq 0$.

To clarify, the gradings in Conjecture 19 are taken $\bmod 2 m$. Thus when $\epsilon$ is $\mathbb{Z}$-graded, the conclusion of Conjecture 19 can be written:

$2 \sum_{k}\left(\operatorname{dim} \operatorname{Hom}^{2 m k}(\epsilon, \epsilon)-\operatorname{dim} B^{2 m k}(\epsilon, \epsilon)\right)-\ell-\chi_{*}(\Lambda)=\sum_{k}\left(2 \operatorname{dim} H^{2 m k} \operatorname{Hom}(\epsilon, \epsilon)-\operatorname{dim} H^{2 m k+1} \operatorname{Hom}(\epsilon, \epsilon)\right)$,

where all gradings are now taken over $\mathbb{Z}$.

Proof of Proposition 21. For convenience, we write $\operatorname{Hom}^{i}(\epsilon, \epsilon)$ as $\operatorname{Hom}^{i}, H^{i} \operatorname{Hom}(\epsilon, \epsilon)$ as $H^{i}$, and $B^{i}(\epsilon, \epsilon)$ as $B^{i}$. Note that $\operatorname{Hom}^{i}$ is generated by the Reeb chords of $\Lambda$ of degree $i-1$, along with $\ell$ generators in degree 0 and $\ell$ generators in degree 1 . Thus

$$
\begin{aligned}
2 \sum_{k} \operatorname{dim} \operatorname{Hom}^{2 m k}-2 \ell-\chi_{*}(\Lambda)= & \sum_{k}(2 k+1)\left(\operatorname{dim} \operatorname{Hom}^{2 m k+2 m}-\operatorname{dim} \operatorname{Hom}^{2 m k}\right) \\
& -\sum_{k}(2 k+1) \sum_{i=0}^{2 m-1}(-1)^{i} \operatorname{dim} \operatorname{Hom}^{2 m k+i+1} \\
= & \sum_{k}(2 k+1) \sum_{i=0}^{2 m-1}(-1)^{i} \operatorname{dim} \operatorname{Hom}^{2 m k+i} \\
= & \sum_{k}(2 k+1) \sum_{i=0}^{2 m-1}(-1)^{i} \operatorname{dim} H^{2 m k+i}+2 \sum_{k} \operatorname{dim} B^{2 m k},
\end{aligned}
$$

where the final equality comes from taking the alternating sum of $\operatorname{dim} \operatorname{Hom}^{i}=\operatorname{dim} B^{i}+\operatorname{dim} H^{i}+\operatorname{dim} B^{i+1}$ from $i=0$ to $i=2 m-1$.

Thus it suffices to show that

$$
\sum_{k}(2 k+1) \sum_{i=0}^{2 m-1}(-1)^{i} \operatorname{dim} H^{2 m k+i}=\sum_{k}\left(2 \operatorname{dim} H^{2 m k}-\operatorname{dim} H^{2 m k+1}\right)-\ell .
$$

But this follows, after a bit of algebraic manipulation, from Sabloff duality ([EES09], cf. [NRS ${ }^{+} 15$, section 5.1.2]), which implies that $\operatorname{dim} H^{i}=\operatorname{dim} H^{2-i}$ unless $i=0$ or $i=2$, and $\operatorname{dim} H^{0}=\operatorname{dim} H^{2}+\ell$. 


\section{REFERENCES}

[Bar94] S. A. Barannikov. The framed Morse complex and its invariants. Adv. Soviet Math., 21:93-115, 1994.

[BC14] Frédéric Bourgeois and Baptiste Chantraine. Bilinearized Legendrian contact homology and the augmentation category. J. Symplectic Geom., 12(3):553-583, 2014.

[BD01] John C. Baez and James Dolan. From finite sets to Feynman diagrams. In Mathematics unlimited-2001 and beyond, pages 29-50. Springer, Berlin, 2001.

[Cha10] Baptiste Chantraine. Lagrangian concordance of Legendrian knots. Algebr. Geom. Topol., 10(1):63-85, 2010.

[Cha13] Baptiste Chantraine. Lagrangian concordance is not a symmetric relation. arXiv:1301.3767, 2013.

[Che02] Yuri Chekanov. Differential algebra of Legendrian links. Invent. Math., 150(3):441-483, 2002.

[CNS] Christopher R. Cornwell, Lenhard Ng, and Steven Sivek. Obstructions to Lagrangian concordance. Algebr. Geom. Topol. To appear.

[CP05] Yu. V. Chekanov and P. E. Pushkar'. Combinatorics of fronts of Legendrian links, and Arnol'd's 4-conjectures. Uspekhi Mat. Nauk, 60(1(361)):99-154, 2005. Translation in Russian Math. Surveys, 60(1):95-149, 2005.

[EES05] Tobias Ekholm, John Etnyre, and Michael Sullivan. Orientations in Legendrian contact homology and exact Lagrangian immersions. Internat. J. Math., 16(5):453-532, 2005.

[EES09] Tobias Ekholm, John B. Etnyre, and Joshua M. Sabloff. A duality exact sequence for Legendrian contact homology. Duke Math. J., 150(1):1-75, 2009.

[EGH00] Y. Eliashberg, A. Givental, and H. Hofer. Introduction to symplectic field theory. Geom. Funct. Anal., (Special Volume, Part II):560673, 2000. GAFA 2000 (Tel Aviv, 1999).

[EHK] Tobias Ekholm, Ko Honda, and Tamás Kálmán. Legendrian knots and exact Lagrangian cobordisms. J. Eur. Math. Soc. (JEMS). To appear.

[Ekh07] Tobias Ekholm. Morse flow trees and Legendrian contact homology in 1-jet spaces. Geom. Topol., 11:1083-1224, 2007.

[Eli98] Yakov Eliashberg. Invariants in contact topology. In Proceedings of the International Congress of Mathematicians, Vol. II (Berlin, 1998), number Extra Vol. II, pages 327-338, 1998.

[ENS02] John B. Etnyre, Lenhard L. Ng, and Joshua M. Sabloff. Invariants of Legendrian knots and coherent orientations. J. Symplectic Geom., 1(2):321-367, 2002.

[EP96] Y. Eliashberg and L. Polterovich. Local Lagrangian 2-knots are trivial. Ann. of Math. (2), 144(1):61-76, 1996.

[FI04] Dmitry Fuchs and Tigran Ishkhanov. Invariants of Legendrian knots and decompositions of front diagrams. Mosc. Math. J., 4(3):707717, 783, 2004.

[Fuc03] Dmitry Fuchs. Chekanov-Eliashberg invariant of Legendrian knots: existence of augmentations. J. Geom. Phys., 47(1):43-65, 2003.

[HR15] Michael B. Henry and Dan Rutherford. Ruling polynomials and augmentations over finite fields. J. Topol., 8(1):1-37, 2015.

[MP07] João Faria Martins and Timothy Porter. On Yetter's invariant and an extension of the Dijkgraaf-Witten invariant to categorical groups. Theory Appl. Categ., 18:No. 4, 118-150, 2007.

[MS05] Paul Melvin and Sumana Shrestha. The nonuniqueness of Chekanov polynomials of Legendrian knots. Geom. Topol., 9:1221-1252 (electronic), 2005

[NRS ${ }^{+}$15] Lenhard Ng, Dan Rutherford, Vivek Shende, Steven Sivek, and Eric Zaslow. Augmentations are sheaves. arXiv:1502.04939, 2015.

[NS06] Lenhard L. Ng and Joshua M. Sabloff. The correspondence between augmentations and rulings for Legendrian knots. Pacific J. Math., 224(1):141-150, 2006.

[NZ09] David Nadler and Eric Zaslow. Constructible sheaves and the Fukaya category. J. Amer. Math. Soc., 22(1):233-286, 2009.

[Rut06] Dan Rutherford. Thurston-Bennequin number, Kauffman polynomial, and ruling invariants of a Legendrian link: the Fuchs conjecture and beyond. Int. Math. Res. Not., pages Art. ID 78591, 15, 2006.

[Sab05] Joshua M. Sabloff. Augmentations and rulings of Legendrian knots. Int. Math. Res. Not., (19):1157-1180, 2005.

[She15] Vivek Shende. Generating families and constructible sheaves. arXiv:1504.01336, 2015.

[STZ14] Vivek Shende, David Treumann, and Eric Zaslow. Legendrian knots and constructible sheaves. arXiv:1402.0490, 2014.

Lenhard NG, Department of Mathematics, Duke University

E-mail address: ng@math.duke.edu

DAN Rutherford, Department of Mathematical SCIEnCEs, Ball State University

E-mail address: rutherford@bsu.edu

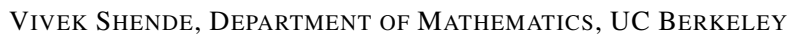

E-mail address: vivek@math. berkeley.edu

Steven Sivek, Department of Mathematics, Princeton University

E-mail address: ssivek@math.princeton. edu 